\title{
Amenability and measure of maximal entropy for semigroups of rational maps.
}

\author{
Carlos Cabrera and Peter Makienko
}

November 8, 2021

\begin{abstract}
In this article we discuss relations between algebraic and dynamical properties of non-cyclic semigroups of rational maps.
\end{abstract}

\section{Introduction}

In a series of works J. Ritt (see [29] and [30]) studied non-trivial relations and functional equations on the semigroup of rational maps. Specifically, Ritt was interested in the solution of equations of the following type

$$
A \circ B=C \circ D
$$

where $A, B, C$ and $D$ are rational maps. Ritt gave solutions to these equations for polynomials but there were obstacles in the case of rational maps. Ritt's theory for rational maps is still under investigation and presents many open questions. The paper 28 contains a short survey on the modern treatment in this area as well of an ample list of references. In particular, the references provided whitin [28] also include a series of Pakovich's works on recent developments of Ritt's theorems for rational maps.

In the paper [13], D. Ghioca, T. J. Tucker and M. E. Zieve proved the following interesting result:

If for polynomials $P$ and $Q$ there exists a point $z_{0} \in \overline{\mathbb{C}}$ such that the intersection of the forward orbits of $z_{0}$, with respect to $P$ and $Q$, is an infinite set then $P$ and $Q$ share a common iterate. That is, there are natural numbers $n, m$ such that $P^{n}=Q^{m}$.

In other words, the dynamical intersection property implies an algebraic equation on $P$ and $Q$.

Our first theorem generalizes the theorem above. Recall that a polynomial (rational map) $Q$ is exceptional if either $\operatorname{deg}(Q)=1$ or $Q$ is affinely (Möbius) conjugated either to $z^{n}$ (with $n \in \mathbb{Z}$ ) or to a Chebyshev polynomial (or a Lattès

\footnotetext{
${ }^{0}$ This work was partially supported by PAPIIT IN106719 and CONACYT CB15/255633. MSC2010: 37F10, 43A07,37F44
} 
map). We say that a family of polynomials (rational maps) $\mathcal{F}$ is non-exceptional if $\mathcal{F}$ contains a non-exceptional polynomial (rational map). Given a family of maps $\mathcal{F}$ we denote by $S(\mathcal{F})$ the semigroup generated by the family $\mathcal{F}$.

Theorem 1. Given a finite non-exceptional family $\mathcal{F}$ of non-injective polynomials. Then, the following conditions are equivalent.

1. For every pair $P, Q \in \mathcal{F}$ there exists a point $z_{0} \in \overline{\mathbb{C}}$ such that

$$
\#\left(\left\{\bigcup P^{j}\left(z_{0}\right) \cap \bigcup Q^{k}\left(z_{0}\right)\right\}\right)=\infty .
$$

2. For every pair $P, Q \in S(\mathcal{F})$ there are integers $m, n$ such that $P^{m}=Q^{n}$.

3. The semigroup $S(\mathcal{F})$ is amenable with $R I M(S(\mathcal{F})) \subset L I M(S(\mathcal{F}))$. Where $R I M(S(\mathcal{F}))$ and $\operatorname{LIM}(S(\mathcal{F}))$ stands for the sets of right and left invariant means respectively.

4. $S(\mathcal{F})$ is embeddable into a virtually cyclic group.

Recall that a virtually cyclic group is a group containing a cyclic subgroup of finite index. As shown in Example 1 below, a semigroup of polynomials may be embeddable into a virtually cyclic group but not into a metacyclic group, those are virtually cyclic groups for which the cyclic subgroup is normal.

For families of rational maps we have the following.

Theorem 2. Let $\mathcal{F}$ be a non-exceptional collection of non-injective rational maps containing an element not Möbius conjugated to a polynomial. Then the following conditions are equivalent.

1. The semigroup $S(\mathcal{F})$ is right amenable and for every pair $P, Q \in S(\mathcal{F})$ there is a point $z_{0} \in \overline{\mathbb{C}}$ such that

$$
\#\left\{\bigcup P^{j}\left(z_{0}\right) \cap \bigcup Q^{k}\left(z_{0}\right)\right\}=\infty .
$$

2. For every pair $P, Q \in S(\mathcal{F})$ there exist natural numbers $m, n$ such that $P^{m}=Q^{n}$.

3. The semigroup $S(\mathcal{F})$ is right amenable and embeddable into a group.

4. The semigroup $S(\mathcal{F})$ is $\rho$-right-amenable, where $\rho$ denotes the Lyubich representation (definitions below), and for every pair $P, Q \in \mathcal{F}$ there is a point $z_{0} \in \overline{\mathbb{C}}$ such that

$$
\#\left(\left\{\bigcup P^{j}\left(z_{0}\right) \cap \bigcup Q^{k}\left(z_{0}\right)\right\}\right)=\infty
$$

If a semigroup of rational maps is finitely generated and satisfies the condition (3) of Theorem 2, then the associated group is virtually cyclic.

The following two theorems describe the right-amenable semigroups of rational maps. 
Theorem 3. Let $\rho$ be the Lyubich representation. Assume $S$ is a $\rho$-rightamenable semigroup of rational maps containing a non-exceptional rational map which is not Möbius conjugated to a polynomial, then the following statements hold true.

1. There exists a probability measure $\mu$ invariant under $S$.

2. If $P \in S$ and $\operatorname{deg}(P)>1$ then $\mu$ is the measure of maximal entropy of $P$.

Since right amenability implies $\rho$-right-amenability for every bounded representation $\rho$ (definitions and discussion in the next section), then Theorem 3 holds for right-amenable semigroups of rational maps.

For polynomials we have the following result.

Theorem 4. Given a finite non-exceptional collection of polynomials $\mathcal{F}$, the following conditions are equivalent.

1. The semigroup $S(\mathcal{F})$ is $\rho$-right-amenable for the Lyubich representation $\rho$.

2. The semigroup $S(\mathcal{F})$ is right amenable.

3. There exists a probability measure $\mu$ invariant under $S(\mathcal{F})$ which coincides with the measure of maximal entropy for every element in $S(\mathcal{F})$.

The equivalence of (1) and (2) is rare even among groups. According to M. Day (see Theorem 2 in [7) a semigroup $S$ is right amenable if and only if $S$ is $\rho$-right amenable for every bounded representation $\rho$.

Hence the theorem above presents an interest from this point of view.

For rational maps, Theorem 4 is still an open question. We propose the following:

Conjecture. If a semigroup $S$ of rational maps admits an invariant probability meausre which is the measure of maximal entropy of every non-injective element of $S$, then $S$ is a right-amenable semigroup.

Theorem 4 gives a partial answer to this conjecture (see also Theorem 20 and Corollary [23).

In fact, Theorems 1 14 hold in more general settings, see the discussion in Section 3.

In the last section we discuss amenability properties for another important representation in holomorphic dynamics, namely Ruelle representation. The Ruelle representation is closely related to quasiconformal deformations of rational maps. In Proposition 37 and Proposition 38 we show that a right amenable semigroup $S$ is quasiconformally deformable whenever $S$ contains a hyperbolic element which admits a non-trivial quasiconformal deformation. Even more, a semigroup $S$ of rational maps is structurally stable whenever $S$ satisfies the Levin relations, is finitely generated and contains a structurally stable element.

To every rational map $R$ of degree at least 2 , we associate a right-amenable finitely generated semigroup of operators $D(R)$ acting on the space $L_{1}(A)$, for 
every Lebesgue measurable subset $A$ completely invariant with respect to $R$. In Proposition 39 we observe that if $A$ does not possess a Beltrami differential, invariant under $R$, then the action of $D(R)$ on $L_{1}(A)$ is left amenable. The main theorem of the last section is the following.

Theorem 5. If $R$ is a rational map and assume that the action of $D(R)$ in $L_{1}(\overline{\mathbb{C}})$ is left-amenable. Then the following conditions are equivalent:

1. The Ruelle operator $R_{*}$ has non-zero fixed points in $L_{1}(\overline{\mathbb{C}})$.

2. $R$ is Möbius conjugated to a flexible Lattés map.

The dynamics of non-cyclic semigroups of rational maps initiated by A. Hinkkanen and G. Martin in [15] is now an active area of research in holomorphic dynamics. Yet another approach is presented in [5] and [12].

In particular, in [15] the authors adapt the Klein-Maskit combination theorem to construct free semigroups of rational maps. The arguments in [15, allow to show the following statement.

If the polynomials $P, Q$ have mutually disjoint filled Julia sets, then there are integers $m, n>0$ such that the semigroup $\left\langle P^{m}, Q^{n}\right\rangle$ is free.

So the semigroup $\langle P, Q\rangle$ contains a free two-generated subsemigroup, which is neither right nor left amenable. This observation is another motivation for considering amenability conditions.

We have the following conjecture:

Conjecture. A finitely generated semigroup $S$ not containing a rank 2 free subsemigroup admits an invariant probability meausre which is the measure of maximal entropy of every non-injective element of $S$.

In this work, we will use standard notations and concepts from holomorphic dynamics which can be found, for instance, in [23].

Let us describe an outline of the article as follows, in section 2, we give some basic facts and notions of amenability of semigroups, holomorphic dynamics, measure or maximal entropy and Ruelle and Lyubich representations. We also introduce three intersection properties for semigroups of rational maps, the comparation between them is one of the main motivations of this work leading to Theorem 1, this theorem is proved in a slightly more general version in section 3 as Theorem 36 .

Theorem 2 is the version of Theorem 1 for arbitrarily semigroups of rational maps containing an non-exceptional map not Möbius conjugated to a polynomial. Theorem 2 is proved in section 3 as Theorem 27.

The proofs of Theorem 1 and Theorem 2 are based upon Theorem 3 and Theorem 4 respectively, which describe (and characterize for polynomials) right amenable semigroups of rational maps. These theorems are proven in section 3 as Theorem 19 and Corollary 26, respectively. The gap between the stronger case of polynomials and arbitrary semigroups rational maps is contained in the conjectures above. So in section 3 , we consequently develop all ingredients and combine them toghether to form the proofs. 
Presenting independent interest, Proposition 33 and Corolary 34 describe quotients and representations of rational semigroups for an equivalence relation motivated by Proposition 11. Also, Theorem 31 and Theorem 35 can be regarded as a characterization of amenable semigroups of polynomials.

Finally, last section is devoted to the proof of Theorem 5 which is presented as Theorem 40.

While this paper was under revision, we learned that some results of this paper were generalized in [26] and later the conjecture about rank 2 free semigroups was answered in [3].

Acknowledgement. The authors would like to thank F. Pakovich for useful discussions, and to the referee for useful remarks and suggestions.

\section{Preliminaries}

\subsection{Semigroup amenability}

Let $S$ be a semigroup and let $L_{\infty}(S)$ be the linear space of bounded complex valued functions equipped with the supremum norm. A continuous linear functional $M$ on $L_{\infty}(S)$ is called a mean if $M$ satisfies the following properties:

1. $M$ is positive, that is, if $\phi \in L_{\infty}(S)$ and $\phi \geq 0$ then $M(\phi) \geq 0$.

2. $\|M\|=M\left(\chi_{S}\right)=1$, where $\chi_{S}$ denotes the characteristic function of $S$.

The right and left actions of $S$ onto itself generate right and left actions on the space $L_{\infty}(S)$ given by the formulas

$$
\begin{aligned}
& r_{s}(\phi)(x)=\phi(x s) \\
& l_{s}(\phi)(x)=\phi(s x)
\end{aligned}
$$

for every $s, x \in S$, and $\phi \in L_{\infty}(S)$, respectively.

These actions induce right and left representations of the semigroup $S$ into the semigroup $\operatorname{End}\left(L_{\infty}(S)\right)$ of linear continuous endomorphisms of $L_{\infty}(S)$ given by $s \mapsto r_{s}$ and $s \mapsto l_{s}$.

The semigroup $S$ is called right amenable, or shortly an RA-semigroup, if there exists a mean which is invariant for the right action of $S$ on $L_{\infty}(S)$ that is $M\left(r_{s}(\phi)\right)=M(\phi)$ for every $s \in S$ and $\phi \in L_{\infty}(S)$. We denote by $R I M(S)$ the set of all right invariant means on the semigroup $S$. Note that $R I M(S)$ is a convex, closed subset of $L_{\infty}^{*}(S)$ which does not contains the 0 functional. Even more $R I M(S)$ is compact in the *-weak topology.

Analogously, $S$ is called left amenable, or an LA semigroup for short, if there is a mean invariant under the left action. We denote by $\operatorname{LIM}(S)$ the set of all left invariant means.

Finally, the semigroup $S$ is called an amenable semigroup, if $R I M(S) \cap$ $\operatorname{LIM}(S) \neq \emptyset$.

Let us mention some basic facts about amenable semigroups. Further details and proofs may be found in the papers of M. Day [6] and [8]. 


\section{Basic Facts on Amenability.}

1. Every abelian semigroup is amenable.

2. Every finite group is amenable and not every finite semigroup is amenable. For example, the finite semigroup $\left\langle a, b: a b=a^{2}=a, b a=b^{2}=b\right\rangle$ is not an LA-semigroup.

3. Every semigroup is a subsemigroup of an amenable semigroup. But, for groups every subgroup of an amenable group is amenable.

4. Given a semigroup $S$, let us consider the antiproduct $*$ on the set $S$ defined by $a * b=b a$. The set $S$ equipped with the product $*$ is a semigroup antiisomorphic to $S$ and thus the space $\operatorname{RIM}(S)$ coincides with $\operatorname{LIM}(S, *)$. In the same way, the left action of $S$ on $L_{\infty}(S)$ is the right action of $(S, *)$ on $L_{\infty}(S)$.

5. Let $M$ be an element of either $\operatorname{LIM}(S)$ or $R I M(S)$. If $M\left(\chi_{S_{0}}\right)>0$ for a subsemigroup $S_{0}<S$. Then $S_{0}$ is itself either an LA or an RA-semigroup, respectively.

6. Let $\phi: S_{1} \rightarrow S_{2}$ be an epimorphism of semigroups, then $S_{2}$ is either $R A$ or $L A$ whenever $S_{1}$ is $R A$ or $L A$, respectively.

Let us consider a weaker version of amenability, namely $\rho$-amenability which was introduced by M. Day in [8].

First, we say that a proper right (left) $S$-invariant subspace $X \subset L_{\infty}(S)$ is called either right or left amenable, again RA or LA for short, if $X$ contains constant functions and there exists a mean $M$ such that, when $M$ is restricted to $X$, it induces a functional which is invariant for either the right or left actions of $S$ on $X$, respectively. In other words $X$ is invariant and admits an invariant functional for the associated action. Note that every semigroup admits an amenable subspace, for example, the subspace of constant functions is always amenable.

Now, let $\rho$ be a bounded homomorphism from $S$ into $\operatorname{End}(B)$, where $B$ is a Banach space, and $\operatorname{End}(B)$ is the semigroup of continuous linear endomorphisms. Given a pair $\left(b, b^{*}\right) \in B \times B^{*}$ consider the function $f_{\left(b, b^{*}\right)} \in L_{\infty}(S)$ given by

$$
f_{\left(b, b^{*}\right)}(s)=b^{*}(\rho(s)(b)) .
$$

Let $Y_{\rho} \subset L_{\infty}(S)$, be the closure of the linear span of the family of functions $\left\{f_{\left(b, b^{*}\right)}\right\}$ for all pairs $\left(b, b^{*}\right) \in B \times B^{*}$. Finally let $X_{\rho}$ be the space generated by $Y_{\rho}$ and the constant functions. Note that $X_{\rho}$ and $Y_{\rho}$ are both right and left invariant.

Definition. We will say that $\rho$ is either $R A$ or $L A$ whenever $X_{\rho}$ is either a right or left amenable subspace of $L_{\infty}(S)$, respectively. Also we will say that $S$ is $\rho$-RA or $\rho$-LA whenever $\rho$ has the respective property. Equivalently, that the $\rho$-action of $S$ on $B$ is either RA or LA, respectively. 
To show that, in general, amenability is different from $\rho$-amenability let us recall Day's theorem (see Theorem 2 in [7]).

Theorem 6 (Day Theorem). A semigroup $S$ is either $R A$ or LA if and only if $S$ is either $\rho$-RA or $\rho$-LA, respectively, for every bounded representation $\rho$.

Roughly speaking, the existence of an invariant functional on a proper subspace does not always implies the existence of an invariant functional on $L_{\infty}(S)$.

For example consider a free group $G$ which is neither RA nor LA. Let $h$ be a homomorphism from $G$ onto a non trivial abelian group $\Gamma$, then the space $h^{*}\left(L_{\infty}(\Gamma)\right) \subset L_{\infty}(G)$ is amenable, where $h^{*}(\phi)=\phi \circ h$ is the pull-back operator. We do not know examples of semigroups which are neither $\rho$-RA nor $\rho$-LA for every bounded representation $\rho$, even in the case when the associated Banach space is infinitely dimensional.

\subsection{Maximal entropy and representations}

In this article we consider two important representations of semigroups of rational maps. Namely, Lyubich and Ruelle representations, these are push-forward actions of rational maps on the spaces $C(\overline{\mathbb{C}})$ and $L_{1}(\overline{\mathbb{C}})$ of continuous and Lebesgue integrable functions on the Riemann sphere $\overline{\mathbb{C}}$, respectively.

Let us first discuss Lyubich representation. Every rational map $R$ induces an operator given by

$$
L_{R}(\phi)(y)=\frac{1}{\operatorname{deg}(R)} \sum_{R(x)=y} \phi(x)
$$

where the sum is taken with multiplicities. The operator $L_{R}$ is a continuous endomorphism of $C(\overline{\mathbb{C}})$ with the unit norm. The operator $L_{R}$ was firstly considered by M. Lyubich in [21, we call $L_{R}$ the Lyubich operator of the rational map $R$. Now we reformulate the main results of [22] as follows:

Theorem 7. For every rational map $R$ with $\operatorname{deg}(R)>1$ there exist an invariant non-atomic probability measure $\mu_{R}$ which represents an invariant functional on $C(\mathbb{C})$ with respect to the Lyubich operator $L_{R}$. The measure $\mu_{R}$ is of maximal entropy, ergodic and unique in the following sense: if an $L_{R}$-invariant functional is generated by a non-atomic measure $\nu$, then $\nu$ is a multiple of $\mu_{R}$.

The support of $\mu_{R}$ coincides with the set $J(R)$, the Julia set of $R$. Observe that the Lyubich operator is well defined for every branched self-covering of the Riemann sphere of finite degree.

Definition. Let $f: \overline{\mathbb{C}} \rightarrow \overline{\mathbb{C}}$ be a branched covering of finite degree. We call the correspondence $\rho: f \mapsto L_{f}$ the Lyubich representation.

Lyubich representation gives a homomorphism from the whole semigroup of finite degree branched self-coverings of $\overline{\mathbb{C}}$ into $\operatorname{End}(C(\overline{\mathbb{C}}))$, the semigroup of continuous linear endomorphisms of the space of continuous functions on $\overline{\mathbb{C}}$. 
Note that the uniqueness statement in Theorem 17 is, in general, false for non-holomorphic branched self-coverings (see discussion after Theorem 16). We call a complex valued measure $\nu$ a Lyubich measure for a semigroup $S$ generated by a collection of finite degree branched self-coverings of $\overline{\mathbb{C}}$ whenever $\nu$ induces an $L_{f}$-invariant functional for every $f \in S$.

Now, let us discuss the Ruelle representation of rational maps.

Definition. Let $R$ be a rational map, then the operator

$$
R_{*}(\phi)(y)=\sum_{R(x)=y} \frac{\phi(x)}{\left(R^{\prime}(x)\right)^{2}}
$$

is called the Ruelle transfer operator or the Ruelle operator .

Ruelle operator acts on the space $L_{1}(\overline{\mathbb{C}})$ with $\left\|R_{*}\right\| \leq 1$. The operator $B_{R}(\phi)=\phi(R) \frac{\overline{R^{\prime}}}{R^{\prime}}$ is called the Beltrami operator, which is a continuous endomorphism of $L_{\infty}(\overline{\mathbb{C}})$ with unitary norm. The space Fix $\left(B_{R}\right)$ of fixed points of $B_{R}$ is called the space of invariant Beltrami differentials of $R$. In other words, the form $\phi(z) \frac{\bar{\partial} z}{\partial z}$ is invariant under the pull-back action of $R$ whenever $\phi \in F i x\left(B_{R}\right)$. By Ahlfors-Bers theorem, the space of invariant Beltrami differentials generates all quasiconformal deformations of the map $R$.

The relevance of Ruelle operator comes from the following lemma (see for example [4] and 24]).

Lemma 8. The Beltrami operator $B_{R}$ is dual to the Ruelle operator $R_{*}$.

Let us note that both the Beltrami and the Ruelle operators can be extended to almost everywhere differentiable branched self-coverings of the Riemann sphere.

\subsection{Relations and functional equations on rational maps}

The following theorem was proven by Ritt in 29] and completed by Eremenko in [11.

Theorem 9. Let $S \subset$ Rat be an abelian semigroup of rational maps. Assume that $S$ contains a non-exceptional element $R$ with $\operatorname{deg}(R) \geq 2$. Then for every pair of elements $P, Q \in S$ with $\operatorname{deg}(P), \operatorname{deg}(Q) \geq 2$ there are numbers $m, n$ such that $P^{m}=Q^{n}$.

Definition. We say that the rational maps $Q, R$ satisfy the Levin relations if

$$
Q \circ R=Q \circ Q
$$

and

$$
R \circ Q=R \circ R .
$$

The following theorem is proved in [19] and 20], we present it as formulated by $\mathrm{H}$. Ye in [31. 
Theorem 10. Two non-exceptional rational maps $Q$ and $R$ share the same measure of maximal entropy if and only if there are numbers $m, n$ such that $Q^{m}$ and $R^{n}$ satisfy the Levin relations.

The following theorem is a consequence of Ritt's results given in [30.

Proposition 11 (Ritt). Let $F, A, B$ be rational maps satisfying the equation

$$
F A=F B,
$$

then either $\operatorname{deg}(F)>\operatorname{deg}(A)=\operatorname{deg}(B)$ or $A$ and $B$ share a common right factor, that is, there are rational maps $X, Y$ and $Z$ such that

$$
A=X \circ Z
$$

and

$$
B=Y \circ Z \text {. }
$$

By Proposition 11$]$ if $Q$ and $R$ satisfy the Levin relations, then $Q$ and $R$ share a right common factor. Moreover, if either $Q$ or $R$ is an indecomposable rational map then the rational maps $X$, and $Y$ given in Proposition 11 must be Möbius transformations. Recall that a map $R$ is called indecomposable if whenever we have the equation $R=P \circ T$ then one of the factors, either $P$ or $T$ must be a Möbius transformation.

Also note that the relations given in Proposition 11 pose an obstacle to the left cancellation property (definitions and discussions are given below).

\subsection{Intersection properties}

Now let us introduce three intersection properties which will be discussed in this work.

Definition (Dynamical intersection property). Let $D I P \subset R a t \times R a t$ be the set consisting of the pairs of rational maps $Q, R$ for which there exists a point $z_{0} \in \overline{\mathbb{C}}$ with

$$
\#\left\{\mathcal{O}_{Q}\left(z_{0}\right) \cap \mathcal{O}_{R}\left(z_{0}\right)\right\}=\infty
$$

where $\mathcal{O}_{R}(z)=\bigcup_{n \geq 0} R^{n}(z)$ denotes the forward orbit of $z$.

Definition (Algebraic intersection property). Let $A I P \subset$ Rat $\times$ Rat be the set of all pairs $(Q, R)$ sharing a common iteration.

Note that $A I P \subset D I P$.

Definition (Ideal intersection property). The semigroup $S$ satisfies the left or right ideal intersection property whenever every pair of principal left or right ideals $I, J$ in $S$ have non-empty intersection. 
The last property is closely related to the problem of embedding a semigroup into a group. That is to specify under what circumstances a given semigroup $S$ is "half" of a group.

Let $\Gamma$ be a countable group with a minimal set of generators $\left\langle\gamma_{1}, \ldots, \gamma_{n}, \ldots\right\rangle$, consider the subset $\Gamma_{+}$of all words in the alphabet $\left\{\gamma_{1}, \ldots \gamma_{n}, \ldots\right\}$. Then $\Gamma_{+}$ forms a countable semigroup which is called the positive part of $\Gamma$. Note that $\Gamma$ is generated by $\Gamma_{+}$and $\left(\Gamma_{+}\right)^{-1}$. A countable semigroup $S$ is embeddable into a group if $S$ is isomorphic to the positive part of a group.

Recall that a semigroup $S$ is left cancellative if for $a, b, c \in S$ the equation $c a=c b$ implies $a=b$. An analogous definition applies for a right cancellative semigroup $S$. For example, every semigroup generated by a set of surjective endomorphisms of a set $A$ is always right cancellative. In particular, every semigroup of rational maps is always right cancellative.

If $S$ is both left and right cancellative, then $S$ is called a cancellative semigroup. For instance, any finitely generated free semigroup $S$ is cancellative and, even more, $S$ is embeddable into a finitely generated free group.

The following theorem due to $\mathrm{O}$. Ore provides sufficient conditions for a semigroup to be embeddable into a group (see [25]).

Theorem 12 (Ore Theorem). Let $S$ be a cancellative semigroup, then $S$ is embeddable into a group whenever $S$ satisfies either the left or right ideal intersection property.

In fact, Ore Theorem does not need the countability condition. As a consequence of Ore Theorem we have that every abelian semigroup $S$ is embeddable into a group if and only if $S$ is cancellative. Hence every abelian semigroup of rational maps is embeddable into a group.

In order to apply Ore Theorem, we need either the right or the left ideal intersection property which is known for RA semigroups (see for example [16]). For sake of completeness we include it in the following lemma.

Lemma 13. If $S$ is an $R A$ semigroup then $S$ satisfies the left ideal intersection property.

Proof. If for $P, Q \in S$ we have $S P \cap S Q=\emptyset$ then for every $r$-mean $\nu$ we have

$$
\nu\left(\chi_{S}\right) \geq \nu\left(\chi_{S P}+\chi_{S Q}\right)=\nu\left(\chi_{S P}\right)+\nu\left(\chi_{S Q}\right)=2 \nu\left(\chi_{S}\right)
$$

which is a contradiction.

The following corollary is an immediate consequence of Ore Theorem and Lemma 13

Corollary 14. A semigroup $S$ of rational maps is embeddable into group whenever $S$ is left cancellative and $R A$. 


\subsection{Ergodic actions}

Given an operator $T$ on a Banach space $X$, the $n$-Cesàro averages of $T$ are the operators $A_{n}(T)$ defined for $x \in X$ by

$$
A_{n}(T)(x)=\frac{1}{n} \sum_{i=0}^{n-1} T^{i}(x) .
$$

An operator $T$ on a Banach space $X$ is called mean-ergodic if $T$ is powerbounded, that is, $\left\|T^{n}\right\| \leq M$ for some number $M$ independent of $n$, and the Cesàro averages $A_{n}(T)(x)$ converge in norm for every $x \in X$.

The following fact can be found, for example, in Krengel's book [18].

Separation principle. An operator $T$ is mean-ergodic if and only if $T$ satisfies the principle of separation of fixed points:

If $x^{*}$ is a fixed point of $T^{*}$, where $T^{*}$ denotes the dual operator of $T$, then there exists $y \in X$ a fixed point of $T$ such that $\left\langle x^{*}, y\right\rangle \neq 0$.

Recall that an operator $T$ acting on a Banach space $\mathcal{B}$ is called weakly almost periodic if $\left\{T^{n}(f)\right\}$ is weakly sequentially precompact for every $f \in \mathcal{B}$. The following theorem is due to I. Kornfeld and M. Lin [17].

Theorem 15. Let $T$ be a positive operator with $\|T\| \leq 1$ acting on $L_{1}(X, \mu)$ space. The operator $T$ is weakly almost periodic if and only if $T$ is mean-ergodic.

\section{Lyubich representation}

We start with the following theorem.

Theorem 16. Let $S$ be a semigroup of branched self-coverings of the sphere. If the Lyubich representation of $S$ is right amenable, then there exists a Lyubich probability measure for $S$.

Proof. Let $\rho: S \rightarrow \operatorname{End}(C(\overline{\mathbb{C}}))$ be the Lyubich representation. Let $\sigma$ be a probability measure. Let $H: C(\overline{\mathbb{C}}) \rightarrow L_{\infty}(S)$ be the map defined for $s \in S$ by

$$
H(\phi)(s)=\int_{\overline{\mathbb{C}}} \rho(s)(\phi(z)) d \sigma(z) .
$$

Since the characteristic function $\chi_{\overline{\mathbb{C}}}$ is a fixed element for every Lyubich operator $\rho(s)$, then the closure of the image of $H$ is a subspace $L_{\infty}(S)$ containing the constant functions on $S$. The space $X=\overline{i m(H)}$ is invariant under the right action of $S$. By assumption $X$ admits a non-zero $r$-invariant mean $L$, then the functional $\ell$ given by

$$
\ell(\phi)=L(H(\phi))
$$

is continuous and positive on $C(\overline{\mathbb{C}})$. Let us show that $\ell$ is invariant with respect to $\rho(S)$.

Indeed, for $t \in S$

$$
r_{t}(H(\phi))(s)=H(\phi)(s t)=\int \rho(s t)(\phi) d \sigma
$$




$$
=\int \rho(s)(\rho(t)(\phi)) d \sigma=H(\rho(t)(\phi))(s) .
$$

Since $L$ is $r$-invariant then

$$
\begin{gathered}
\ell(\phi)=L(H(\phi))=L\left(r_{t}(H(\phi))\right) \\
=L(H(\rho(t)(\phi)))=\ell(\rho(t)(\phi)) .
\end{gathered}
$$

By the Riesz representation theorem there exists a probability measure $\mu$ satisfying $\ell(\phi)=\int \phi d \mu$, since $\ell$ is $\rho(t)$ invariant then $\mu$ is a Lyubich measure.

The measure $\mu$ depends on the choice of the measure $\sigma$. For instance, consider $\sigma=\delta_{z_{0}}$ the delta measure on a suitable point $z_{0} \in \overline{\mathbb{C}}$. If the cardinality of $\mathcal{O}_{-}(S)\left(z_{0}\right)$ is finite then $\mu$ is atomic and, in fact, is a linear combination of delta measures based on $\overline{\mathcal{O}_{-}(S)\left(z_{0}\right)}$, where

$$
\mathcal{O}_{-}(S)\left(z_{0}\right)=\bigcup_{n \geq 0, s \in S} s^{-n}\left(z_{0}\right) .
$$

If $S<P o l$ is a polynomial semigroup then, choosing $z_{0}=\infty$, the measure $\delta_{\infty}$ is an atomic Lyubich measure.

If $\overline{\mathcal{O}_{-}(S)\left(z_{0}\right)}$ is infinite then $\mu$ may be non-atomic, as in the case of cyclic semigroups of rational maps.

Even more, for semigroups of non-holomorphic branched coverings of the sphere a non-atomic Lyubich measure may not be unique even for cyclic semigroups. For example, if $f$ is a formal mating of two polynomials, say $P$ and $Q$, then the conformal copies of the measures of maximal entropy for $P, Q$ and $z^{\operatorname{deg} P}$ generate a three dimensional space of Lyubich measures for $f$. One can use the tuning procedure to construct a map with two dimensional space of Lyubich measures, hence by repeating the procedures of mating and tuning we can produce a multidimensional space of Lyubich measures. The definition of tuning and matings was given by A. Douady in section 2 and section 3 of [9].

From Theorem 16 we have the following corollary.

Corollary 17. Let $S$ be an RA semigroup of branched self-coverings of the Riemann sphere. Then there exists a Lyubich probability measure for $S$.

For semigroups of rational maps we have Theorem 3 which gives a stronger conclusion. For this we need the following.

Definition. We say that a collection of rational maps $\mathcal{F}$ is called admissable if it contains a non exceptional element $g$ and either

- $g$ is not Möbius conjugated to a polynomial, or

- $g$ is conjugated to a polynomial but there exists another element $g_{1} \in \mathcal{F}$ such that there is no Möbius map $\gamma$ simultaneously conjugating $g$ and $g_{1}$ to polynomials, or 
- $\mathcal{F}$ consists of rational maps simultaneously Möbius conjugated to a family of polynomials $\mathcal{F}^{\prime}$ and there exists a disk $D_{\mathcal{F}}$ centered at $\infty$ such that $P\left(D_{\mathcal{F}}\right) \subset D_{\mathcal{F}}$ for every $P \in \mathcal{F}^{\prime}$.

In other words, a family $\mathcal{F}$ containing a non exceptional map is admissable if either there is no point $z_{0} \in \overline{\mathbb{C}}$ with $R^{-1}\left(z_{0}\right)=\left\{z_{0}\right\}$ for every $R \in \mathcal{F}$, or, otherwise, there exists a common $R$-invariant topological disk around such $z_{0}$ for every $R \in \mathcal{F}$.

Every finite collection of non-linear polynomials containing a non-exceptional polynomial is admissable, even more every collection of monic non-linear polynomials with bounded coefficients and containing a non-exceptional polynomial is admissable. Finally, a collection $\mathcal{F}$ is admissable if and only if the semigroup $S(\mathcal{F})$ is also admissable. Indeed, if $\mathcal{F}$ is admissable, then $S(\mathcal{F})$ is also admissable. Reciprocally, if $S(\mathcal{F})$ is admissable but $\mathcal{F}$ is not then $\mathcal{F}$ is Möbius conjugated to polynomials and there is no disk $D$ such that $P(D) \subset D$ for all $P \in S(\mathcal{F})$ which contradicts $S(\mathcal{F})$ is admissable.

We also need the following lemma.

Lemma 18. Let $R$ be a non-injective rational map and $\nu$ be a probability Lyubich measure for $R$. Then $\nu=s \mu_{R}+(1-s) \omega$ with $s \in[0,1]$ where $\mu_{R}$ is the measure of maximal entropy and either $\omega=\delta_{a}$ with $R^{-1}(a)=a$ or $\omega=t \delta_{a}+(1-t) \delta_{b}$ with $t \in[0,1]$ and $R^{-1}(\{a, b\})=\{a, b\}$.

In other words, an $L_{R}$-invariant functional is presented by an atomic measure $\nu$ if and only if either $R$ or $R^{2}$ is Möbius conjugated to a polynomial and the support of $\nu$ contains the point associated to $\infty$ as an atom of $\nu$.

Proof. Let us observe that the support of a measure $\nu$ is a completely invariant closed set. Indeed, let $A=\operatorname{supp}(\nu)$ and $\chi_{A}$ be its characteristic function. Since $\int \chi_{A} d \nu=\int L_{R}\left(\chi_{A}\right) d \nu$ and $L_{R}\left(\chi_{A}\right) \leq \chi_{A} \nu$-almost everywhere, then $L_{R}\left(\chi_{A}\right)=\chi_{A} \nu$-almost everywhere. Hence $R^{-1}(A)=A$.

Now, if

$$
W=F(R) \backslash[\text { \{periodic points }\} \cup\{\text { rotational domains }\}]
$$

then $A \cap W=\emptyset$. Otherwise, there is a point $z_{0} \in A \cap W$ and a disk $D$ centered at $z_{0}$ with $\nu(D)>0$ so that $R^{-n}(D)$ forms a pairwise disjoint family of open subsets of $W$, for $n=1,2,3, \ldots$ But this contradicts that $\nu$ is invariant. Since $R^{-1}(A \cap F(R))=A \cap F(R)$, then $A \cap F(R)$ consists of periodic points and, by a normal families argument, contains at most two points $\{a, b\}$. Then $\omega$, which is the atomic part of $\nu$, consists of delta measures based on the set $\{a, b\}$.

If $A \cap J(R)=\emptyset$, then we are done. Otherwise, $A \cap J(R)=J(R)$ and, by Theorem 7 , the restriction of $\nu$ on $J(R)$ is a multiple of the measure of maximal entropy which finishes the proof.

Theorem 19. Let $\mathcal{F}$ be an admissable family of rational maps. Assume $S=$ $S(\mathcal{F})$ admits a right amenable Lyubich representation, then there exists a unique non-atomic Lyubich measure $m_{S}$. Even more, $m_{S}=m_{s}$ for any $s \in S$ with $\operatorname{deg}(s)>1$ where $m_{s}$ is the measure of maximal entropy of $s$. 
Proof. By Theorem 16 and Lemma 18 there is a Lyubich measure $m_{S}$ given by a measure $\sigma=\delta_{y_{0}}$ for some $y_{0} \in \overline{\mathbb{C}}$. Since $\mathcal{F}$ contains a non-exceptional map $g$, then by Lemma 18, the measure $m_{S}=\alpha\left(y_{0}\right) m_{g}+\beta\left(y_{0}\right) \delta_{z_{0}}, \alpha\left(y_{0}\right)+\beta\left(y_{0}\right)=1$ for a suitable $z_{0} \in \overline{\mathbb{C}}$ and $m_{g}$ is the measure of maximal entropy of $g$. If $\beta\left(y_{0}\right) \neq 0$ the $z_{0}$ is determined by the condition $g^{-1}\left(z_{0}\right)=z_{0}$.

If $\alpha\left(y_{0}\right) \neq 0$ for a $y_{0} \in \overline{\mathbb{C}}$, we are done by Theorem $\mathbf{7}$, since $m_{g}$ is the claimed measure.

Let us show that $\alpha\left(y_{0}\right) \neq 0$ for some $y_{0}$. Otherwise $\beta\left(y_{0}\right)=1$ for every $y_{0} \in \overline{\mathbb{C}}$ and $\mathcal{F}$ consists of polynomials, since by conjugation we can assume that $z_{0}=\infty$ because $s^{-1}\left(z_{0}\right)=z_{0}$ for every $s \in S$. Hence $\mathcal{F}$ satisfies the third case of the definition of admissability.

Thus, there exists a disk $D_{\mathcal{F}}$ such that $P\left(D_{\mathcal{F}}\right) \subset D_{\mathcal{F}}$ for every $P \in S$. Let $K=\overline{\mathbb{C}} \backslash D_{\mathcal{F}}$ and take a compact subdisk $D \subset D_{\mathcal{F}}$ centered at $\infty$ and consider $\phi$ a continuous function such that $\phi(z)$ is 1 for $z \in K$ and 0 for $z \in D$.

Hence

$$
\int_{\overline{\mathbb{C}}} \phi(z) d m_{S}(z)=0
$$

Let $x_{0} \in K$, since $s^{-1}(K) \subset K$ for any $s \in S$, then, by construction of $\phi$, for every $s \in S$ we have

$$
H(\phi)(s)=\int_{\overline{\mathbb{C}}} \rho(s)(\phi(z)) d \delta_{x_{0}}(z)=(\rho(s)(\phi))\left(x_{0}\right)=1,
$$

where $\rho$ is the Lyubich representation. Thus $H(\phi)$ is the constant function 1 , hence $M(H(\phi))=1$ for every mean $M$. Besides, for a suitable $r$-invariant mean $L$ we have

$$
L(H(\phi))=\ell(\phi)=\int_{\overline{\mathbb{C}}} \phi(z) d m_{S}(z)=\phi(\infty)=0,
$$

which is a contradiction.

Let $R$ be a rational map with $\operatorname{deg}(R) \geq 2$, then we define $E(R)$ to be the set of all rational maps $Q$ such that

$$
L_{Q}^{*}\left(m_{R}\right)=m_{R}
$$

where $L_{Q}^{*}$ is the dual operator to the Lyubich operator $L_{Q}$ and $m_{R}$ is the measure of maximal entropy of $R$. The set $E(R)$ is a semigroup under composition.

Define $G(R)=E(R) \cap M o b$. Also, $G(R)$ can be characterized as the maximal subgroup of $E(R)$.

Theorem 20. Let $P$ be a non-exceptional polynomial of degree at least 2, and $S$ be a subsemigroup of $E(P)$ then $S$ is right amenable.

This theorem is reciprocal of Theorem 19. We start with the following.

Let $U$ and $T$ be semigroups with a homomorphism $\rho: T \rightarrow \operatorname{End}(U)$. Let $U \rtimes_{\rho} T$ denote the semidirect product of the semigroups $U$ and $T$ which is the set $U \times T$ equipped with the following multiplication

$$
\left(u_{1}, t_{1}\right) \cdot\left(u_{2}, t_{2}\right)=\left(u_{1} \cdot \rho\left(t_{1}\right)\left(u_{2}\right), t_{1} t_{2}\right)
$$


Next proposition puts together two results of M. Klawe (see [16]).

Proposition 21. Let $U$ and $T$ be semigroups and $\rho: T \rightarrow E n d(U)$ be a homomorphism.

- If $U$ and $T$ are $R A$, then $U \rtimes_{\rho} T$ is $R A$.

- If $U$ and $T$ are amenable semigroups and $\rho(t)$ is surjective for every $t \in T$, then $U \rtimes_{\rho} T$ is amenable.

Proof. These appear as Proposition 3.10 and Corollary 3.11 in [16].

Theorem 22. Let $P$ be a polynomial map such that $E(P)$ is not abelian, then there are an abelian subsemigroup $\Gamma<E(P)$ and a homomorphism $\Phi: \Gamma \rightarrow$ $\operatorname{End}(G(P))$ such that the semidirect product $G(P) \rtimes_{\Phi} \Gamma$ is isomorphic to $E(P)$. Moreover, if $P$ is not Möbius conjugated to $z^{n}$ then $\Gamma$ can be chosen as a cyclic semigroup.

Proof. First assume that $P$ is not affinely conjugated to $z^{n}$ for some $n$. Since the elements of $G(P)$ leave the Julia set $J(P)$ invariant, then $G(P)$ is a finite group of rotations around a common center (see for example Lemma 4 of [2]).

Let $Q \in E(P)$ be a non-linear polynomial of minimal degree. Since $E(P)$ is not abelian then by Atela-Hu theorem in [1 for every element $\tilde{Q} \in E(P)$ there is $n \geq 0$ and a $\gamma \in G(P)$ such that $\tilde{Q}=\gamma \circ Q^{n}$. But the degree of $Q$ is minimal and $Q \circ \gamma \in E(P)$ then $Q \circ \gamma=\gamma^{\prime} \circ Q$. Thus the correspondence $\gamma \mapsto \gamma^{\prime}$ defines a homomorphism $\Phi$ from $\langle Q\rangle$ to $\operatorname{End}(G(P))$. With $\Phi$, construct the semidirect product $G(P) \rtimes_{\Phi}\langle Q\rangle$ so the correspondence $(u, g) \mapsto u \circ g$ defines a surjective isomorphism $G(P) \rtimes_{\Phi}\langle Q\rangle \rightarrow E(P)$.

Now assume $P$ is affinely conjugated to $z^{n}$ for some $n$. Then $E(P)$ is not abelian and after a suitable conjugation $G(P)$ becomes $G\left(z^{n}\right)$ which is generated by the group of all rotations around 0 and the element $1 / z$. In this situation, we can choose a subsemigroup $\Gamma \subset E(P)$ conjugated to all powers of $z$. Therefore $\Gamma$ is an abelian infinitely generated semigroup acting on $G(P)$ by semiconjugacy as a semigroup of surjective endomorphisms of $G(P)$. Again, the correspondence $(u, T) \rightarrow u \circ T$ generates an isomorphism $G(P) \rtimes_{\Phi} \Gamma \rightarrow E(P)$.

As an immediate consequence we have.

Corollary 23. Let $P$ be a polynomial, then $E(P)$ is RA. Even more, if $P$ is conjugated to $z^{n}$ then $E(P)$ is amenable.

Proof. If $E(P)$ is abelian then it is amenable. Otherwise, the corollary follows from Proposition 21 and Theorem 22 .

Given a rational map $R$, let

$$
\operatorname{Deck}(R)=\{\gamma \in M o b: R \circ \gamma=R\}
$$

and

$$
\operatorname{Aut}(R)=\{\gamma \in M o b: R \circ \gamma=\gamma \circ R\} .
$$


Corollary 24. Let $P$ be a polynomial with finite $G(P)$. Let $Q \in E(P)$ be a non-injective polynomial of minimal degree. Then there exist natural numbers $m, n$ such that $G(P)$ is generated by $\operatorname{Deck}\left(Q^{m}\right)$ and $A u t\left(Q^{n}\right)$.

Proof. By Theorem 22, the map $Q$ defines an endomorphism $\Phi(Q): G(P) \rightarrow$ $G(P)$ by the semiconjugacy $Q \circ \gamma=\Phi(Q)(\gamma) \circ Q$. Since $G(P)$ is finite the map $\Phi(Q)$ is surjective if and only if $\Phi(Q)$ is an automorphism of $G(P)$. In this case, there exists $n$ such that $\Phi^{n}(Q)=I d$ and thus $Q^{n} \circ \gamma=\Phi^{n}(Q)(\gamma) \circ Q=\gamma \circ Q^{n}$.

If $\Phi(Q)$ is not an automorphism, then as $G(P)$ is finite there exists $k$ so that $\Phi$ acts on $\Phi^{k}(G(P))$ as an automorphism and

$$
G(P) \simeq \operatorname{Ker}\left(\Phi^{k}\right) \otimes \operatorname{Im}\left(\Phi^{k}\right) .
$$

Let $m>0$ be the minimal number satisfying the equation above. Let $n$ be the minimal natural number such that $\Phi^{n}: \operatorname{Im}\left(\Phi^{m}\right) \rightarrow \operatorname{Im}\left(\Phi^{m}\right)$ is identity. Then every $\gamma \in \operatorname{Im}\left(\Phi^{m}\right)$ commutes with $Q^{n}$. On the other hand, if $\gamma \in \operatorname{Ker}\left(\Phi^{m}\right)$ then $Q^{m}(\gamma)=Q^{m}$.

Example 1. Let $P(z)=z^{5}+z^{2}$, then

$$
G(P)=\left\{\lambda z: \lambda^{3}=1\right\}
$$

and

$$
E(P)=\left\{\lambda^{k} P^{l}, \text { for } k=0,1,2 \text {, and } l=0,1, \ldots\right\}
$$

is amenable by Theorem 22 ,

Since $G(P)=A u t\left(P^{2}\right)$ then by Corollary 24 we have $m=0, n=2$. Therefore, the polynomial $Q=\lambda P$ commutes with $P^{2}$ but does not commutes with $P$. In particular, amenability does not implies commutativity even for polynomials.

Lemma 25. Let $P$ be a polynomial with finite $G(P)$, then there exists $\mu \in$ $R I M(E(P))$ so that $\mu\left(\chi_{\langle Q\rangle}\right)>0$ for every $Q \in E(P)$.

Proof. We follow Klawe's proof of Proposition 21 (Proposition 3.10 in [16]). We summarize Klawe's construction of a RIM for a semidirect product $S=U \rtimes_{\rho} T$ of RA semigroups $U$ and $T$ with representation $\rho: T \rightarrow \operatorname{End}(U)$ as follows.

- Choose both a RIM $\phi$ for $U$ and a RIM $\nu$ for $T$.

- For each $f \in L_{\infty}(S)$ construct the function $\tilde{f} \in L_{\infty}(T)$ as follows: given $a \in T$ let $f_{a}(u)=f(u, a)$ for $u \in U$, then the family of functions $\left\{f_{a}\right\}$ belongs to $L_{\infty}(U)$. Let $\tilde{f}(a)=\phi\left(f_{a}\right)$.

- The mean $\mu$ on $S$ given by

$$
\mu(f)=\nu(\tilde{f})
$$

is a RIM for $S$. 
By Theorem 22, the semigroup $E(P)$ contains a polynomial map $R$ such that $E(P)$ is isomorphic to the semidirect product of $G(P)$ and $\langle R\rangle$. Choose two RIMs $\phi$ and $\nu$ for $G(R)$ and $\langle R\rangle$, respectively. Since $G(R)$ is finite, $\phi\left(\chi_{A}\right) \geq$ $\frac{1}{|G(P)|}$ for each subset $A \subset G(P)$. Let $Q \in E(P)$ then there exists a number $m \geq 0$ such that, for every $n>0, Q^{n}=\gamma_{n} R^{m n}$ with $\gamma_{n} \in G(P)$.

If $f=\chi_{\langle Q\rangle}$ is the characteristic function of $\langle Q\rangle$ in $L_{\infty}(E(P))$, then the family of functions $f_{R^{n}}(\gamma)=f\left(\gamma R^{n}\right)$ belongs to $L_{\infty}(G(P))$. Thus the function $\tilde{f}\left(R^{n}\right)=\phi\left(f_{R^{n}}\right) \in L_{\infty}(\langle R\rangle)$. By construction $\tilde{f} \geq \frac{1}{|G(P)|} \chi_{\left\langle R^{m}\right\rangle}$. Since $\nu$ is finitely additive and $r$-invariant, we conclude that $\mu(f)=\nu(\tilde{f}) \geq \frac{1}{|G(P)| m}>$ 0 .

Now we are ready to prove Theorem 20.

Proof of Theorem [20. Let $Q \in S<E(P)$ with $Q \neq I d$. By Corollary 23 the semigroup $E(P)$ is RA. By Lemma 25, there exists $\mu \in R I M(E(P))$ such that $\mu\left(\chi_{\langle Q\rangle}\right)>0$. Hence $\mu\left(\chi_{S}\right) \geq \mu\left(\chi_{Q}\right)>0$. We finish the proof by applying property 5 in Section 2.1 Let $S_{0}$ be a subsemigroup of $S$, if there is $\mu \in R I M(S)$ such that $\mu\left(\chi_{S_{0}}\right)>0$, then $S_{0}$ is right amenable itself.

The following Corollary implies the proof of Theorem 4

Corollary 26. For an admissable collection of polynomials $\mathcal{P}$ the following conditions are equivalent.

1. The semigroup $S(\mathcal{P})$ is right amenable.

2. The semigroup $S(\mathcal{P})$ is Lyubich right amenable.

3. There exist $P \in \mathcal{P}$ such that $\mathcal{P} \subset E(P)$.

Proof. Part (1) implies (2) by Theorem 6. Part (2) implies (3) by Theorem 19. Part (3) implies (1) by Theorem 20.

Theorem 27. For an admissable collection $\mathcal{F}$ of non-injective rational maps, the following affirmations are equivalent.

1. The semigroup $S(\mathcal{F})$ is $R A$ and $S(\mathcal{F}) \times S(\mathcal{F}) \subset D I P$.

2. $S(\mathcal{F}) \times S(\mathcal{F}) \subset A I P$.

3. The semigroup $S(\mathcal{F})$ is $R A$ and embeddable into a group.

To prove Theorem 27 we need the following several facts.

Theorem 28. Let $S=\left\langle s_{1}, \ldots, s_{m}\right\rangle$ be a finitely generated right cancellative semigroup satisfying the Levin relations $s_{i} \circ s_{j}=s_{i}^{2}$ for all $i, j$. Then

- The semigroup $S$ is right amenable.

- If $S$ is left amenable then $S$ is cyclic. 
Proof. For the first point, the proof uses standard ergodic arguments. Let $m$ be the number of generators of $S$.

By Levin relations we have the following dichotomy for any pair of elements $s_{i}$ and $s_{j}$ in the generating set.

Namely either

$$
s_{i}=s_{j}
$$

or

$$
\left\langle s_{i}\right\rangle \cap\left\langle s_{j}\right\rangle=\emptyset .
$$

Indeed, if there are numbers $k, q$ such that $s_{i}^{k}=s_{j}^{q}$ then by the Levin relations $k=q$ and $s_{i}^{k+1}=s_{j}^{k+1}$ but by right cancellation we have $s_{i}=s_{j}$.

Let $\mathcal{L}(s)$ be the length function that is the infimum of the length of $s$ as a word in the letters $\left\{s_{1}, \ldots, s_{m}\right\}$. Since every element of $S$ is the iteration of a generator, then $\#\{s: \mathcal{L}(s) \leq n\}=m n+1$.

The semigroup $S$ acts by the right on $L_{\infty}(S)$. The spherical average $\Theta$ of this action is given by

$$
\Theta_{n}(\phi)=\frac{1}{m n+1} \sum_{\mathcal{L}(s) \leq n} r_{s}(\phi) .
$$

Note that for each $n$ the operator $\Theta_{n}$ is positive with $\left\|\Theta_{n}\right\|=1$ and $\Theta_{n}\left(\chi_{S}\right)=\chi_{S}$, where $\chi_{S}$ is the characteristic function on $S$.

We claim that if $h$ is a generator of $S$ then

$$
\left\|r_{h}\left(\Theta_{n}(\phi)\right)-\Theta_{n}(\phi)\right\| \leq \frac{2 m\|\phi\|}{m n+1}
$$

for every $\phi \in L_{\infty}(S)$. Indeed, by the Levin relations for the right action of $S$ we have

$$
r_{h}\left(\Theta_{n}(\phi)\right)-\Theta_{n}(\phi)=\frac{1}{m n+1}\left[\sum_{i=1}^{m} r_{s_{i}}^{n+1}(\phi)-\sum_{s_{i} \neq h} r_{s_{i}}(\phi)-\phi\right],
$$

but the right action is a contraction, that is $\left\|r_{h}\right\| \leq 1$ so the claim follows.

Let $\mathcal{M}$ be an $L_{1}$ mean on $L_{\infty}(S)$, that is $\mathcal{M}$ is induced by a non-negative function $\omega: S \rightarrow \mathbb{C}$ with $L_{1}$-norm $\|\omega\|=\sum_{s \in S} \omega(s)=1$ and $\mathcal{M}(\phi)=$ $\sum_{s \in S} \phi(s) \omega(s)$. Consider the family of means $\mathcal{M}_{n}=\Theta_{n}^{*}(\mathcal{M})$ where $\Theta_{n}^{*}$ is the dual operator of $\Theta_{n}$. Then $\mathcal{M}_{n}$ forms a precompact family in the $*$-weak topology. Note that $\mathcal{M}_{n}\left(\chi_{S}\right)=1$ since $\Theta\left(\chi_{S}\right)=\chi_{S}$, so we get that any accumulation point of $\left\{\mathcal{M}_{n}\right\}$ is a mean. If $\mathcal{M}_{0}$ is an accumulation point then by the claim $\mathcal{M}_{0}$ is invariant by the right action of any generator of $S$. Hence $\mathcal{M}_{0} \in R I M(S)$. Which finishes the first part of the proof.

The last part is by contradiction. Assume that $S=\left\langle s_{1}, \ldots, s_{m}\right\rangle$ is left amenable with $m>1$ and $\left\{s_{i}\right\}_{i=1}^{m}$ forms a minimal set of generators. By the dichotomy above, $S=\bigsqcup_{i=1}^{m}\left\langle s_{i}\right\rangle$. Take $L \in L I M(S)$, since $L\left(\chi_{S}\right)=1$ there exists a generator $s_{i}$ such that $L\left(\chi_{\left\langle s_{i}\right\rangle}\right)>0$. As $m>1$, let $s_{j}$ be a generator 
with $i \neq j$ and let $\Gamma=\left\langle s_{i}, s_{j}\right\rangle$ then $L\left(\chi_{\Gamma}\right)=L\left(\chi_{\left\langle s_{i}\right\rangle}+\chi_{\left\langle s_{j}\right\rangle}\right)>0$. By Fact 5 of the Basic Facts of amenability, the semigroup $\Gamma$ is a non-cyclic LA semigroup with two generators.

Let us show that $\left\langle s_{i}\right\rangle \cap\left\langle s_{j}\right\rangle \neq \emptyset$. Indeed, if $\left\langle s_{i}\right\rangle \cap\left\langle s_{j}\right\rangle=\emptyset$ then by Levin relations $s_{j} \circ s \in\left\langle s_{j}\right\rangle$ for every $s \in \Gamma$. Hence

$$
l_{s_{j}}\left(\chi_{\left\langle s_{i}\right\rangle}\right)(s)=\chi_{\left\langle s_{i}\right\rangle}\left(s_{j} \circ s\right)=0 .
$$

By left invariance $L\left(\chi_{\left\langle s_{i}\right\rangle}\right)=L\left(l_{s_{j}}\left(\chi_{\left\langle s_{i}\right\rangle}\right)\right)=0$. Which contradicts the choice of $s_{i}$. Hence, $\left\langle s_{i}\right\rangle \cap\left\langle s_{j}\right\rangle \neq \emptyset$. Therefore, by the dichotomy above, $s_{i}=s_{j}$ which is again a contradiction with the choice of $s_{j}$.

Theorem 29. Let $S=S(\mathcal{F})$ be an amenable semigroup satisfying $R I M(S) \subset$ $\operatorname{LIM}(S)$, where $\mathcal{F}$ is an admissable collection of rational maps. Then, for every $f$ and $h$ in $S$ with $\operatorname{deg}(f), \operatorname{deg}(h)>1$ there are numbers $m, n$ satisfying $f^{m}=h^{n}$.

Proof. Fix two arbitrary elements $f, h \in S$ with $\operatorname{deg}(f), \operatorname{deg}(h)>1$. Then by Theorem 19 the maps $f$ and $h$ have the same measure of maximal entropy, and by Theorem 10 there are iterates $F$ and $H$ of $f$ and $h$ respectively, satisfying the Levin relations. Let $\Gamma=\langle F, H\rangle$ be the semigroup generated by $F$ and $H$. By Theorem 28 the semigroup $\Gamma$ is RA. If $\Gamma$ is LA, then again by Theorem 28 $F=H$ and we are done.

Let us show that indeed $\Gamma$ is a left amenable semigroup. We follow a Theorem of Granirer (see Theorem E2 in [14) aswell as the arguments of the proof of this theorem. The theorem states:

Let $S$ be an LA semigroup with left cancellation and let $S_{0} \subset S$ be an $L A$ subsemigroup. Then there is a linear isometry $T$ from the subspace of left invariant elements of $L_{\infty}^{*}\left(S_{0}\right)$ into the subspace of left invariant elements of $L_{\infty}^{*}(S)$ with $T\left(\operatorname{LIM}\left(S_{0}\right)\right) \subset \operatorname{LIM}(S)$.

More precisely, using the left cancellation and the left cosets of $S_{0}$ in $S$, Granirer constructs an isometric linear section $j: L_{\infty}\left(S_{0}\right) \rightarrow L_{\infty}(S)$ to the restriction $\rho: L_{\infty}(S) \rightarrow L_{\infty}\left(S_{0}\right)$, which is a positive linear map, so that for every left invariant functional $\nu_{0} \in L_{\infty}^{*}\left(S_{0}\right)$ the following formula holds (page 55 of [14]).

$$
T\left(\nu_{0}\right)(x)=\nu_{0}(\rho(x))
$$

for every $x \in j\left(L_{\infty}\left(S_{0}\right)\right)$.

Let $s \in S_{0}$ and $r_{s}, \tilde{r}_{s}$ be the right action of $s$ on $L_{\infty}(S)$ and $L_{\infty}\left(S_{0}\right)$ respectively, then for every $x \in L_{\infty}(S)$ we have

$$
\rho\left(r_{s}(x)\right)=\tilde{r}_{s} \rho(x) .
$$

Now assume $T\left(\nu_{0}\right) \in R I M(S)$ and $r_{s}\left(j\left(x_{0}\right)\right)-j\left(\tilde{r}_{s}\left(x_{0}\right)\right) \in \operatorname{ker}\left(T\left(\nu_{0}\right)\right)$ for every $x_{0} \in L_{\infty}\left(S_{0}\right)$, then $\nu_{0} \in R I M\left(S_{0}\right)$. Indeed

$$
\nu_{0}\left(\tilde{r}_{s}\left(x_{0}\right)\right)=T\left(\nu_{0}\left(j\left(\tilde{r}_{s}\left(x_{0}\right)\right)\right)\right)=T\left(\nu_{0}\right)\left(r_{s}\left(j\left(x_{0}\right)\right)\right)=T\left(\nu_{0}\right)\left(j\left(x_{0}\right)\right)=\nu_{0}\left(x_{0}\right) .
$$


Suppose $T\left(\nu_{0}\right) \in R I M(S)$ then we claim

$$
r_{s}\left(j\left(x_{0}\right)\right)-j\left(\tilde{r}_{s}\left(x_{0}\right)\right) \in \operatorname{ker}\left(T\left(\nu_{0}\right)\right) .
$$

Indeed, let $f=j\left(\chi_{S_{0}}\right)$, then

$$
T\left(\nu_{0}\right)(f)=\nu_{0}(\rho(f))=\nu_{0}\left(\chi_{S_{0}}\right)=1 .
$$

Moreover, $T\left(\nu_{0}\right)\left(\chi_{S}\right)=1$ thus $\chi_{S}-f \in \operatorname{ker}\left(T\left(\nu_{0}\right)\right)$. But $T\left(\nu_{0}\right)$ is a positive functional and $\chi_{S}-f \geq \chi_{S \backslash \sup (f)}$, then $\phi \in \operatorname{ker}\left(T\left(\nu_{0}\right)\right)$ whenever $\operatorname{supp}(\phi) \in$ $S \backslash \operatorname{supp}(f)$. Since $j$ is a positive isometric section of the restriction map $\rho$, then for every $s \in S_{0}$ and $x_{0} \in L_{\infty}\left(S_{0}\right)$ we have

$$
\operatorname{supp}\left(r_{s}\left(j\left(x_{0}\right)\right)-j\left(\tilde{r}_{s}\left(x_{0}\right)\right)\right) \subset S \backslash \operatorname{supp}\left(j\left(\chi_{S_{0}}\right)\right)
$$

as claimed.

By assumption $T\left(\nu_{0}\right) \in \operatorname{LIM}(S)$ whenever $\nu_{0} \in \operatorname{LIM}\left(S_{0}\right)$. Hence, by the claim if $\operatorname{LIM}(S) \subset R I M(S)$ then $\nu_{0} \in R I M\left(S_{0}\right)$ and, in particular, $\operatorname{LIM}\left(S_{0}\right) \subset$ $\operatorname{RIM}\left(S_{0}\right)$.

To apply Granirer Theorem and the discussion above, we consider $S^{*}$ to be the semigroup $S$ endowed with the antiproduct. Since $S$ is amenable with right cancellation and $\operatorname{RIM}(S) \subset L I M(S)$, then $S^{*}$ is an amenable semigroup with left cancellation and $\operatorname{LIM}\left(S^{*}\right) \subset R I M\left(S^{*}\right)$, hence $\Gamma^{*} \subset S^{*}$ is left amenable. It follows that $\Gamma^{*}$ is an RA semigroup and hence $\Gamma$ is left amenable. This finishes the proof.

Let us note that as a corollary we have the following statement.

Corollary 30. Let $S$ be a right cancellative amenable semigroup satisfying $R I M(S) \subset L I M(S)$. Consider an $R A$ subsemigroup $S_{0}<S$, then $R I M\left(S_{0}\right) \subset$ $\operatorname{LIM}\left(S_{0}\right)$.

Theorem 31. Assume $\mathcal{F} \times \mathcal{F} \subset A I P$ for a non-exceptional collection of rational maps $\mathcal{F}$, then $S=S(\mathcal{F})$ is amenable.

Proof. The proof of the theorem is a consequence of a theorem due to M. Day [6] which states: Let $S=\bigcup S_{n}$, where $S_{n}$ are semigroups such that for every $m, n$ there exists $k$ with $S_{m} \cup S_{n} \subset S_{k}$. Then $S$ is amenable whenever the semigroups $S_{n}$ are amenable for every $n$.

Fix a non-exceptional element $Q \in \mathcal{F}$. For $n>0$, let $S_{n}$ be the subsemigroup of all elements in $S$ commuting with $Q^{n}$, then by assumption $S=\bigcup_{n} S_{n}$ and moreover for every finite collection of indexes $n_{1}, \ldots, n_{k}$ there exists $N$ such that $\bigcup_{i=1}^{k} S_{n_{i}} \subset S_{N}$, for instance, take $N=\prod n_{i}$. To finish the proof we have to show that the semigroups $S_{n}$ are amenable.

Indeed, for each $n$ let

$$
M_{n}(Q)=\left\{\mu \in L_{\infty}^{*}\left(S_{n}\right): l_{Q^{n}}^{*}(\mu)=r_{Q^{n}}^{*}(\mu)=\mu, \mu \geq 0, \mu\left(\chi_{S_{n}}\right)=\|\mu\|=1\right\} .
$$

$M_{n}(Q)$ is a non-empty, closed, convex and compact set with respect to the *-weak topology of $L_{\infty}^{*}\left(S_{n}\right)$. In fact, $M_{n}(Q)$ is a subset of the unit sphere in 
$L_{\infty}^{*}\left(S_{n}\right)$ so does not contains the zero element. Fix $n$, since every element $s \in S_{n}$ commutes with $Q^{n}$ then $r_{s}^{*}$ and $l_{s}^{*}$ leave $M_{n}(Q)$ invariant. Hence we constructed left and right representations $\rho_{l}$ and $\rho_{r}$ of $S_{n}$ into the semigroup $\operatorname{End}\left(M_{n}\right)$ of continuous endomorphisms of $M_{n}$.

By Theorem 9 every element of $S_{n}$ shares a common iteration with $Q^{n}$, thus the images $\Gamma_{l}:=\operatorname{Im}\left(\rho_{l}\right)$ and $\Gamma_{r}:=\operatorname{Im}\left(\rho_{r}\right)$ are groups in $\operatorname{End}\left(M_{n}\right)$.

If $\Gamma_{l}$ and $\Gamma_{r}$ are finite groups then $S_{n}$ is amenable for every $n$. Indeed, as $M_{n}$ is convex the averages defined by $A_{r}(\nu)=\frac{1}{\#\left\{\Gamma_{r}\right\}} \sum_{\gamma \in \Gamma_{r}} \gamma(\nu)$ leaves $M_{n}$ invariant. This means that $A_{r}\left(M_{n}\right)=R I M\left(S_{n}\right)$. Similarly, if $A_{l}(\nu)=$ $\frac{1}{\# \Gamma_{l}} \sum_{\gamma \in \Gamma_{l}} \gamma(\nu)$ then $A_{l}\left(M_{n}\right)=\operatorname{LIM}\left(S_{n}\right)$. But we have $A_{r}^{2}=A_{r}, A_{l}^{2}=A_{l}$ and $A_{r} \circ A_{l}=A_{l} \circ A_{r}$ then $A_{r} \circ A_{l}\left(M_{n}\right) \subset \operatorname{LIM}\left(S_{n}\right) \cap R I M\left(S_{n}\right)$. Thus $S_{n}$ is amenable.

To finish, we need the following result of F. Pakovich ([28):

Let $f$ be a non-exceptional rational map of degree at least 2 , let $C(f)$ be the semigroup of all rational maps commuting with $f$. Then there are finitely many rational maps $x_{1}, \ldots, x_{k} \in C(f)$ such that every $g \in C(f)$ has the form $g=x_{i} \circ f^{l}$ for some $i$ and $l \geq 0$.

Hence $\Gamma_{r}$ and $\Gamma_{l}$ belong to the image of a finite set of elements, so these groups are indeed finite.

In the proof of the previous theorem, it is enough that $\Gamma_{l}$ and $\Gamma_{r}$ are amenable. While the preparation of this work, Pakovich kindly inform us about his theorem in [28] which significantly shortened our original proof of Theorem 31 .

We are ready to prove Theorem 27

Proof of Theorem 27. Let us show that (1) implies (2). Since $S$ is RA, then by Theorem 10 and Theorem 19 for every $P$ and $Q$ in $\mathcal{F}$ there are numbers $m$ and $n$ such that $P^{m} \circ Q^{n}=P^{2 m}$ and $Q^{n} \circ P^{m}=Q^{2 n}$. Let us show that $P^{m}=Q^{n}$. Indeed, the pair $\left(P^{m}, Q^{n}\right)$ satisfies the intersection property. So, there exist $z_{0}$ and sequences $k_{i}, l_{i}$ such that $P^{m k_{i}}\left(z_{0}\right)=Q^{n l_{i}}\left(z_{0}\right)$.

First assume that $k_{i}=l_{i}$, then by the Levin relations we have

$$
Q^{n} \circ Q^{n\left(k_{i}-1\right)}\left(z_{0}\right)=P^{m} \circ P^{m\left(k_{i}-1\right)}\left(z_{0}\right)=P^{m} \circ Q^{n\left(k_{i}-1\right)}\left(z_{0}\right)
$$

So $P^{m}$ and $Q^{n}$ coincide on the infinite set $\left\{Q^{n\left(k_{i}-1\right)}\left(z_{0}\right)\right\}$, thus $P^{m}=Q^{n}$.

If $k_{i} \neq l_{i}$, then again using the Levin relations we obtain

$$
P^{m\left(k_{i}+1\right)}\left(z_{0}\right)=P^{m} \circ Q^{n l_{i}}\left(z_{0}\right)=P^{m\left(l_{i}+1\right)}\left(z_{0}\right)
$$

hence $z_{0}$ has a finite orbit, which contradicts that $(P, Q) \in D I P$. For a nonexceptional $Q \in \mathcal{F}$ and for every $P \in \mathcal{F}$ there exists $n$ such that $P$ commutes with $Q^{n}$. So, for every element $R \in S$ there exists a number $m=m(R)$ such that $R$ commutes with $Q^{m}$. Thus $\left\langle R, Q^{m}\right\rangle$ is abelian and, by Theorem 9 , $R$ and $Q$ share a common iteration. Therefore, every pair of elements in $S$ also share a common iteration.

Theorem 31 gives the implication from (2) to (1). 
Now, let us show the equivalence of (2) and (3).

First let us show that (3) implies (2). Since $S$ is RA, by Theorem 10 and Theorem [19, if $R$ and $Q$ are non-identity elements in $S$, then there exist numbers $n$ and $m$ such that $R^{m}$ and $Q^{n}$ satisfy the Levin relations but then $R^{m}=Q^{n}$ since $S$ is embeddable into a group.

(2) implies (3). First let us show that $S$ is a cancellative semigroup. We already know that $S$ is right cancellative, so let us show that is also left cancellative. Assume there exist three elements $A, X, Y$ in $S$ with $A X=A Y$. Then $P=X A$ and $Q=Y A$ satisfy the Levin relations

$$
\begin{aligned}
& P \circ Q=P \circ P \\
& Q \circ P=Q \circ Q .
\end{aligned}
$$

By assumption there are numbers $m$ and $n$ such that $P^{m}=Q^{n}$ then $m=n$ since $\operatorname{deg}(P)=\operatorname{deg}(Q)$. Again by the Levin relations $P^{m+1}=Q^{m+1}$ then

$$
X A=P=Q=Y A
$$

and $X=Y$ by right-cancellation. By Lemma 13 $S$ has the left ideal intersection property. Now we have fullfilled the conditions of Ore Theorem which finishes the proof.

Remark. Let us note that in the proof of Theorem 27 it is enough that the intersection $\mathcal{O}_{+}\left(P^{m}, z_{0}\right) \cap \mathcal{O}_{+}\left(Q^{n}, z_{0}\right)$ is sufficiently large. For instance, if

$$
\#\left\{\mathcal{O}_{+}\left(P^{m}, z_{0}\right) \cap \mathcal{O}_{+}\left(Q^{n}, z_{0}\right)\right\}>\operatorname{deg}\left(P^{m}\right) \operatorname{deg}\left(Q^{n}\right)
$$

the arguments still follow. Since bounds are invariants of the semigroup, it is interesting to find precise bounds on the intersection of the orbits.

Another conclusion that follows from Theorem 27 is that $r$-amenability is necessary to compare the intersection property with the algebraic property of sharing a common iterate. As an immediate corollary we have.

Corollary 32. Let $R$ be a non exceptional rational map and let $S(R)$ be the semigroup of rational maps commuting with $R$, then $S(R)$ is an embeddable semigroup.

Proof. Let $\Gamma(R)=S(R) \cap M o b$ then $S(R)$ is generated by $\Gamma(R)$ and $S(R) \backslash \Gamma(R)$. By Theorem 9 and Theorem [31, the semigroup $S(R) \backslash \Gamma(R) \subset A I P$ and is amenable. Since $\Gamma(R)$ is a finite group, it is amenable. Hence $S(R)$ is amenable because it is the disjoint union of amenable semigroups. By Corollary 14 it is enough to show that $S(R)$ is left cancellative. But by Theorem 9 the semigroup $S(R) \backslash \Gamma(R)$ is a semigroup satisfying the conditions of Theorem 27part (2), so $S(R) \backslash \Gamma(R)$ is cancellative.

Finally, if there exist $Q \in S(R) \backslash \Gamma(R)$ and $a, b \in \Gamma(R)$ with $a \neq b$ and $Q \circ a=Q \circ b$. This implies that $\operatorname{Deck}(Q)$ contains $a \circ b^{-1}$ which belongs to $\Gamma(R)$. Then $Q$ and $a b^{-1} Q$ satisfies the Levin relations, since $Q$ and $a b^{-1} Q$ share a common iterate then $a b^{-1}=I d$ which is a contradiction. 
In the theorems above we used the equation $X \circ A=X \circ B$ to study leftcancellation of the semigroups. The maps $P=A \circ X$ and $Q=B \circ X$ satisfy the Levin relations and, by Theorem [10, $P$ and $Q$ have the same measure of maximal entropy. In [27, Pakovich proved the reciprocal theorem, that is:

If $P \circ Q=P \circ P$ and $Q \circ P=Q \circ Q$ then there are rational maps $X, A, B$ such that $P=A \circ X$ and $Q=B \circ X$ and $X \circ A=X \circ B$.

So, if $P$ and $Q$ satisfy the Levin relation then by Pakovich theorem above we arrive to the equation $X \circ A=X \circ B$.

Definition. Given a semigroup $S<$ Rat we say that $A \approx B$ if there exists $X \in G$ so that $X \circ A=X \circ B$.

In general, relation $\approx$ is not an equivalence relation. However, for instance, if $S$ satisfies the left ideal intersection property, then $\approx$ is an equivalence relation in $S$.

It is interesting to characterize when the equation $X \circ A=X \circ B$ defines an equivalence relation on semigroups of rational maps.

Proposition 33. Let $S=S(\mathcal{F})$ be an $R A$ semigroup, where $\mathcal{F}$ is an admissable collection of rational maps. Then the relation $\approx$ defines an equivalence relation and the quotient semigroup $S_{1}=S / \approx$ is embeddable into a group. Even more, if $\pi: S \rightarrow S_{1}$ is a projection homomorphism, then for every $P, Q \in S$ with $\operatorname{deg}(P), \operatorname{deg}(Q) \geq 2$ there are numbers $m$ and $n$ so that $\pi(P)^{m}=\pi(Q)^{n}$.

Proof. The proof that $\approx$ is an equivalence relation relies on standard amenability arguments (see for example [16]).

To verify that $\approx$ is an equivalence relation, it is enough to check transitivity. Indeed, assume that there are $a, b, c \in S$ such that $a \approx b$ and $b \approx c$, thus there are $f_{1}, f_{2} \in S$ with $f_{1} a=f_{1} b$ and $f_{2} b=f_{2} c$. By Lemma13, the semigroup $S$ has the left ideal intersection property, so there are $\alpha, \beta \in S$ such that $\alpha f_{1}=\beta f_{2}$, thence

$$
\alpha f_{1} a=\alpha f_{1} b=\beta f_{2} b=\beta f_{2} c=\alpha f_{1} c .
$$

The multiplication induced over representative classes endows $S_{1}=S / \approx$ with a semigroup product. By Fact 6 of the Basic Facts of amenability, $S_{1}$ is an RA semigroup. In particular, $S_{1}$ has the left ideal intersection property. To show that $S_{1}$ is embbedable into a group, by Ore Theorem we need to verify that $S_{1}$ is cancellative. First $S_{1}$ is $r$-cancellative since $S$ is also $r$-cancellative and, by construction, $S_{1}$ is $l$-cancellative.

Finally, since $S$ is RA, by Theorem 19 and Theorem 10, for every $P, Q \in S$ there are numbers $m, n$ such that $P^{m}$ and $Q^{n}$ satisfy the Levin relations. Since $S$ is embeddable into a group then $\pi\left(P^{m}\right)=\pi\left(Q^{n}\right)$ as claimed.

The following corollary produces, in the polynomial case, a realization for semigroups of the type of $S_{1}$ in the proposition above.

Corollary 34. Let $S=S(\mathcal{F})$ be an $R A$ semigroup, where $\mathcal{F}$ is an admissable collection of polynomials, then there exists a polynomial $P$ and an isomorphism $\phi: S / \approx \rightarrow E(P)$. 
Proof. Since $S$ is RA, by Theorem [19, Theorem 20] and Theorem 22] there exist a polynomial $P$, such that $S \subset E(P)$, and numbers $r, s$ and $t$ so that every element $Q \in S$ has the form $Q=\gamma \circ h \circ P^{t}$ where $\gamma \in \operatorname{Deck}\left(P^{s}\right)$ and $h \in \operatorname{Aut}\left(P^{r}\right)$. Thus the class $[Q]$ contains a unique element $h \circ P^{t}$ then the correspondence $[Q] \mapsto h \circ P^{t}$ induces the desired representation.

For arbitrary semigroups of rational maps, the previous corollary is still an open question.

For polynomials we have the following theorem.

Theorem 35. Let $\mathcal{F}$ be a non-exceptional family of polynomials with $\mathcal{F} \times \mathcal{F} \subset$ $A I P$. Then the semigroup $S(\mathcal{F})$ is amenable with $R I M(S(\mathcal{F})) \subset L I M(S(\mathcal{F})$ ).

Proof. Let $P \in \mathcal{F}$ be non-exceptional, then by the conditions we have $S(\mathcal{F}) \subset$ $E(P)$. If $E(P)$ is abelian, then $S(\mathcal{F})$ is abelian and hence $S(\mathcal{F})$ is amenable with $R I M(S(\mathcal{F}))=\operatorname{LIM}(S(\mathcal{F}))$. Otherwise, by Theorem 22, there is a polynomial $T \in E(P)$, a finite group $G(P)=E(P) \cap M o b$ and a representation $\rho:\langle T\rangle \rightarrow$ $\operatorname{End}(G(P))$ by semiconjugation so that $E(P) \cong G(P) \rtimes_{\rho}\langle T\rangle$. By Corollary 24. there are numbers $r, s$ such that the group $G(P)$ is the direct product of $K(P)=\operatorname{ker}\left(\rho\left(T^{r}\right)\right)$ and $A(P)=A u t\left(T^{s}\right)$. Let $A E(P)$ be the subsemigroup of $E(P)$ generated by $T$ and $A(P)$.

We claim that every subsemigroup $\Gamma$ in $A E(P)$ is amenable with $R I M(\Gamma) \subset$ $L I M(\Gamma)$.

To prove the claim. First,

$$
\sum_{\gamma \in A(P)} r_{\gamma}(\psi)=\sum_{\gamma \in A(P)} l_{\gamma}(\psi)
$$

for every $\psi \in L_{\infty}(A E(P))$.

Indeed if $s \in A E(P)$ then $s=h \circ T^{k}$ for a suitable $h \in A(P)$ and $k \geq 0$. Since $\rho(T)$ is an automorphism of $A(P)$, then

$$
\begin{gathered}
\sum_{\gamma \in A(P)} r_{\gamma}(\psi)(s)=\sum_{\gamma \in A(P)} \psi\left(h \circ T^{k} \circ \gamma\right) \\
=\sum_{\gamma \in A(P)} \psi\left((\rho(T))^{k}(\gamma) \circ h \circ T^{k}\right)=\sum_{\gamma \in A(P)} l_{\gamma}(\psi)\left(h \circ T^{k}\right) .
\end{gathered}
$$

Then for every $\phi \in R I M(A E(P))$, the averages

$$
A_{r}=\frac{1}{\#\{A(P)\}} \sum_{\gamma \in A(P)} r_{\gamma}
$$

and

$$
A_{l}=\frac{1}{\#\{A(Q)\}} \sum_{\gamma \in A(P)} l_{\gamma},
$$

satisfy

$$
\phi=A_{r}^{*}(\phi)=A_{l}^{*}(\phi)
$$


Since $l_{\gamma}^{*} \circ A_{l}^{*}=A_{l}^{*} \circ l_{\gamma}^{*}=A_{l}^{*}$, we conclude that $l_{\gamma}^{*} \phi=\phi$ for every $\gamma \in A(P)$. In other words, every right invariant mean $\phi$ is invariant by the left action of $A(P)$.

Second, let us show that $l_{T}^{*}(\phi)=\phi$.

For every $\psi \in L_{\infty}(A E(P))$ we have

$$
A_{l}\left(l_{T}(\psi)\right)(s)=A_{l}\left(r_{T}(\psi)\right)(s) .
$$

Indeed, let $s=h \circ T^{k}$ then

$$
\begin{gathered}
A_{l}\left(\psi\left(T \circ h \circ T^{k}\right)\right)=A_{l}\left(\psi\left[(\rho(T))(h) \circ h^{-1}\left(h \circ T^{k} \circ T\right)\right]\right) \\
=A_{l}\left(\psi\left(h \circ T^{k} \circ T\right)\right)=A_{l}\left(r_{T}(\psi)\right)(s) .
\end{gathered}
$$

By duality and the fact that $\phi$ is left invariant under $A(P)$, we get

$$
l_{T}^{*}\left(A_{l}^{*}(\phi)\right)=r_{T}^{*}\left(A_{l}^{*}(\phi)\right)=\phi
$$

which implies $l_{T}^{*}(\phi)=\phi$. Hence, by above $\phi \in L I M(A E(P))$.

Third, let $\Gamma<A E(P)$, then $\Gamma$ is right cancellative and by Corollary 23 the semigroup $\Gamma$ is RA. Then by Corollary 30 we have $\operatorname{RIM}(\Gamma) \subset \operatorname{LIM}(\Gamma)$, as claimed.

To finish the proof of the theorem, we have to show that $S(\mathcal{F})$ is isomorphic to a subsemigroup of $A E(P)$.

Let $Q \in \mathcal{F}$ be a polynomial of minimal degree. Then $Q$ has the following expression.

$$
Q=h \circ \gamma \circ T^{l}
$$

for a suitable $l \geq 1$ and $h \in K(P)$ and $\gamma \in A(P)$. Fix $m \leq r$ such that $h \in \operatorname{Ker}\left(\rho\left(T^{m}\right)\right)$ and put

$$
\tilde{h}=h \circ \rho\left(T^{l}\right)(h) \circ \rho\left(T^{2 l}\right) \ldots \circ \rho\left(T^{(m-1) l}\right)(h) .
$$

Then $\tilde{h}^{-1} \circ Q \circ \tilde{h}=\gamma \circ T^{l}$. The family $\tilde{\mathcal{F}}=\tilde{h}^{-1} \circ \mathcal{F} \circ \tilde{h}$ generates a semigroup $S(\tilde{\mathcal{F}})$ isomorphic to $S(\mathcal{F})$. Note that $\tilde{Q}=\tilde{h}^{-1} \circ Q \circ \tilde{h}=\gamma \circ T^{l} \in \tilde{\mathcal{F}}$.

Now let us show that $S(\tilde{\mathcal{F}})<A E(P)$. It is enough to show that $\tilde{\mathcal{F}} \subset A E(P)$. Otherwise, assume that $\tilde{\mathcal{F}}$ contains a polynomial $R=\alpha \circ \beta \circ T^{t}$ for $t \geq 1$, $\alpha \in K(P) \backslash\{I d\}$ and $\beta \in A(P)$. By assumption there are numbers $d, e>0$ such that $R^{e}=\tilde{Q}^{d}$, hence $R$ commutes with $\tilde{Q}^{d}$. If $i=\#\{K(P)\}$ and $j=\#\{A(P)\}$ then for $k=i j d$ we have

$$
R \circ \tilde{Q}^{k}=\alpha \circ \beta \circ \rho\left(T^{l}\right)(\gamma) \circ T^{t} \circ T^{k l}=\tilde{Q}^{k} \circ R=\gamma \circ \beta \circ T^{k l} \circ T^{t} .
$$

Then $\alpha \in A(P)$ which is a contradiction by Corollary 24 ,

Theorem 36. Given an admissable collection of polynomials $\mathcal{F}$, the following statements are equivalent.

1. $\mathcal{F} \times \mathcal{F} \subset D I P$. 
2. $\mathcal{F} \times \mathcal{F} \subset A I P$.

3. $S(\mathcal{F}) \times S(\mathcal{F}) \subset D I P$.

4. $S(\mathcal{F}) \times S(\mathcal{F}) \subset A I P$.

5. $S(\mathcal{F})$ is amenable with $\operatorname{RIM}(S(\mathcal{F})) \subset L I M(S(\mathcal{F}))$.

6. The semigroup $S(\mathcal{F})$ is embeddable into a virtually cyclic group.

Proof. By the Ghioca-Tucker-Zieve Theorem in [13] (1) is equivalent to (2) and (3) is equivalent to (4). Clearly (3) implies (1). By Theorem 29 (5) implies (4). Finally, by Theorem 35 (2) implies (5).

Now (4) implies (6). By Theorem 27t the semigroup $S(\mathcal{F})$ is embeddable into a group. As in the proof of Theorem 35 there exist a polynomial $T$ and a finite group $A(T) \subset M o b$ so that $T$ acts on $A(T)$ by semiconjugacy and generates a representation $h:\langle T\rangle \rightarrow A u t(A(T))$. The semigroup $A E(T)=\langle T, A(T)\rangle \cong$ $A(T) \rtimes_{h}\langle T\rangle$ contains an isomorphic copy of $S(\mathcal{F})$. Let us show that $S(\mathcal{F})$ is a subsemigroup of a virtually cyclic group. First note that $A E(T) \cong A(T) \rtimes_{\tilde{h}} \mathbb{N}$ where $\tilde{h}(n)=h\left(T^{n}\right) \in \operatorname{Aut}(A(T))$. Since $\tilde{h}(n)$ is an automorphism we can extend $\tilde{h}$ on negative integers by the formula

$$
\tilde{h}(-n)=\left(h\left(T^{n}\right)\right)^{-1} .
$$

Hence $A(T) \rtimes_{\tilde{h}} \mathbb{N} \subset A(T) \rtimes_{\tilde{h}} \mathbb{Z}$. But $A(T) \rtimes_{\tilde{h}} \mathbb{Z}$ is a semidirect product of a cyclic group with a finite group, so it is virtually cyclic, then $A E(T)$ is the positive part of a virtually cyclic group.

Now (6) implies (4). Assume that $S(\mathcal{F})$ is embeddable into a virtually cyclic group $\Gamma$, and $\tau$ be the generator of the corresponding cyclic subgroup of finite index. Let $T$ be an element in $S(\mathcal{F})$ corresponding to $\tau$, let $P \in S(\mathcal{F})$ of degree at least 2 and $p \in \Gamma$ the corresponding element. Then $p$ is an element of infinite order, so there exists $k$ with $p^{k} \in\langle\tau\rangle$, hence $(P, T) \in A I P$.

Now we proceed to the proof of Theorem [5 for which we devote the last section.

\section{Left amenability of Ruelle representation}

We begin with the following observation. We say that a semigroup $S<$ Rat is deformable if there exists $f: \overline{\mathbb{C}} \rightarrow \overline{\mathbb{C}}$ a quasiconformal homeomorphism so that $S_{f}=f \circ S \circ f^{-1}<R a t$ and $S_{f}$ is not Möbius conjugated to $S$.

Proposition 37. Let $S$ be an $R A$ semigroup of non-injective rational maps. If $S$ contains a hyperbolic structurally stable map then $S$ is deformable.

Proof. Let $R$ be a hyperbolic structurally stable element of $S$, then by Theorem 19 for every $Q \in S$ with $\operatorname{deg}(Q)>1$ we have $J(Q)=J(R)$. By the Levin relations we have 
- Every $Q \in S$ with $\operatorname{deg}(Q)>1$ is hyperbolic.

- For every periodic component $D$ in the Fatou set $F(R)=\overline{\mathbb{C}} \backslash J(R)$ we have

$$
Q^{-1}\left(\mathcal{O}_{-}(R, D)\right)=\mathcal{O}_{-}(R, D)
$$

for every $Q \in S$ and where $\mathcal{O}_{-}(R, D)=\bigcup_{n=0}^{\infty} R^{-n}(D)$.

Let $K_{D}: \mathcal{O}_{-}(R, D) \rightarrow \mathbb{C}$ be the König linearizing function $D$ for $R$ in $\mathcal{O}_{-}(R, D)$, so $K_{D}(R)=\lambda K_{D}$ for some multiplier $\lambda$. Note that $K_{D}$ also linearizes every $Q \in S$ with $\operatorname{deg}(Q)>1$. Indeed, by the Levin relations we have numbers $m$ and $n$ so that $R^{n} \circ Q^{m}=R^{n} \circ R^{n}$ and $Q^{m} \circ R^{n}=Q^{m} \circ Q^{m}$ then $K_{D}\left(Q^{m}\right)=$ $\lambda^{n} K_{D}$. Then the differential $\mu=\overline{\frac{K_{D}^{\prime}}{K_{D}} K_{D}^{\prime}} \frac{d \bar{z}}{d z}$ is invariant for $Q$ and $R$, so $\mu$ is an invariant Beltrami differential for every element in $S$. Thus, for $t \in(0,1)$, let $g_{t}$ be the quasiconformal map with Beltrami coefficient $t \mu$. Then $g_{t}$ defines a non-trivial deformation for $S$.

Remark. Let us note the following curious fact, if the semigroup $S$ is quasiconformally deformable with Beltrami differential $\mu$ such that $\overline{\operatorname{supp}(\mu)} \neq$ $\overline{\mathbb{C}}$, then for all $R, Q \in S$ with $\operatorname{deg}(R), \operatorname{deg}(Q)>1$ we have $J(R)=J(Q)$.

For a subclass of RA semigroups we can say more. Let $S<R a t$ be a semigroup and let $\phi: S \rightarrow$ Rat be an monomorphism preserving the degree, that is $\operatorname{deg}(\phi(Q))=\operatorname{deg}(Q)$ for every $Q \in S$. We will say that $S$ is structurally stable if every monomorphism preserving degree $\phi: S \rightarrow$ Rat, which is sufficiently close to the identity on generators, is generated by a quasiconformal homeomorphism of $\overline{\mathbb{C}}$.

Proposition 38. Let $\mathcal{F}=\left\{R_{i}\right\}$ be a finite collection of rational maps of degree at least 2 such that $R_{i} \circ R_{j}=R_{i}^{2}$ for every pair $i, j$. Then the semigroup $S(\mathcal{F})$ is structurally stable whenever $S(\mathcal{F})$ contains a structurally stable map.

Proof. If $g \in S(\mathcal{F})$ is structurally stable, then $g$ is indecomposable and therefore is one of the generators, say $R_{1}$. If $\phi: S(\mathcal{F}) \rightarrow$ Rat is a representation sufficiently closed to the identity representation, there exist a quasiconformal homeomorphism $f: \overline{\mathbb{C}} \rightarrow \overline{\mathbb{C}}$ such that $\phi\left(R_{1}\right)=f \circ R_{1} \circ f^{-1}$.

We claim that $\phi(Q)=f \circ Q \circ f^{-1}$ for every $Q \in S(\mathcal{F})$.

It is enough to check the latter equality holds for the generators $R_{i}$. If $\mu=\frac{\bar{\partial} f}{\partial f}$, then $\mu$ is invariant for all generators by the Levin relations.

First let us assume that $R_{i}=\gamma_{i} \circ R_{1} \circ \gamma_{i}^{-1}=\gamma_{i} \circ R_{1}$ and $\phi\left(R_{i}\right)=h_{i} \circ \phi\left(R_{1}\right) \circ$ $h_{i}^{-1}$ for suitables $h_{i} \in \operatorname{Deck}\left(\phi\left(R_{1}\right)\right)$ and $\gamma_{i} \in \operatorname{Deck}\left(R_{1}\right)$, respectively. Since $\gamma_{i}$ leaves $\mu$ invariant then $\operatorname{Deck}\left(\phi\left(R_{1}\right)\right)=f \circ \operatorname{Deck}\left(R_{1}\right) \circ f^{-1}$. If the semigroup $T=\left\langle S(\mathcal{F}), \operatorname{Deck}\left(R_{1}\right)\right\rangle$ then $\phi(S(\mathcal{F})) \subset f \circ T \circ f^{-1} \subset$ Rat.

As $\phi\left(R_{i}\right)$ is close to $R_{i}$ for all $i$, it follows that $h_{i}$ is close to $\gamma_{i}$ and $\gamma_{i}$ is close to $f \circ \gamma_{i} \circ f^{-1}$, as $f$ is close to the identity too.

Therefore, $h_{i}, f \circ \gamma_{i} \circ f^{-1} \in \operatorname{Deck}\left(\phi\left(R_{1}\right)\right)$ are sufficiently close and hence coincide since $\operatorname{Deck}\left(\phi\left(R_{1}\right)\right)$ is discrete. In conclusion, $\phi(S(\mathcal{F}))=f \circ S(\mathcal{F}) \circ f^{-1}$ as claimed. 
It remains to show that $R_{i}=\gamma_{i} \circ R_{1} \circ \gamma_{i}^{-1}=\gamma_{i} \circ R_{1}$ and $\phi\left(R_{i}\right)=h_{i} \circ \phi\left(R_{1}\right) \circ$ $h_{i}^{-1}$ for suitables $h_{i} \in \operatorname{Deck}\left(\phi\left(R_{1}\right)\right)$ and $\gamma_{i} \in \operatorname{Deck}\left(R_{1}\right)$ for every $i$.

Since $R_{1} \circ R_{i}=R_{1}^{2}$ hence $\operatorname{Deck}\left(R_{i}\right) \subset \operatorname{Deck}\left(R_{1}\right)$ then by Theorem 9 the maps $R_{1}$ and $R_{i}$ share a common right factor, that is there are rational maps $X, Y$ and $W$ such that $R_{1}=X \circ W$ and $R_{i}=Y \circ W$. But $R_{1}$ is indecomposable then $\operatorname{Deg}(X)=\operatorname{Deg}(Y)=1$. It follows that $X \circ Y^{-1} \in \operatorname{Deck}\left(R_{1}\right)$ and $R_{i}=$ $Y \circ X^{-1} \circ R_{1}$. Finally, the map $\phi\left(R_{1}\right)$ is structurally stable as a quasiconformal deformation of a structurally stable map, so it is also indecomposable. Now we can repeat the arguments for $\phi\left(R_{1}\right)$.

Therefore, a semigroup $S$ satisfying the Levin relations possesses an non-zero invariant Beltrami differential if and only if there is an element of $S$ possessing an invariant Beltrami differential.

In what follows, for every rational map $R$ and a every completely invariant set $A \subset \overline{\mathbb{C}}$ of positive Lebesgue measure, we construct a semigroup of operators satisfying the Levin relations and acting on $L_{1}(A)$ and show that the action is left amenable whenever $R$ does not admits a non-zero Beltrami differential supported on $A$.

Definition. Let $R$ be a rational map. Let $\sigma$ be an analytic arc in $\mathbb{C}$ containing all critical values of $R$. Let $U=\overline{\mathbb{C}} \backslash \sigma$ and $D=R^{-1}(U)$, then $D=\bigcup_{i=1}^{d e g(R)} D_{i}$ and $\pi_{1}\left(D_{i}\right)=1$ and $R: D_{i} \rightarrow U$ is holomorphic homeomorphism. Set $R_{i}=\left.R\right|_{D_{i}}$ and for each $i, j$ define the piecewise conformal map

$$
h_{i, j}= \begin{cases}R_{j}^{-1} \circ R_{i}, & \text { on } D_{i} \\ R_{i}^{-1} \circ R_{j}, & \text { on } D_{j} \\ I d, & \text { otherwise. }\end{cases}
$$

Then $h_{i, j}$ is a piecewise conformal almost everywhere bijection such that $h_{i, j}^{2}=$ $I d$ and $h_{i, i}=I d$ everywhere. We denote by $D(R)$ the group generated by the maps $h_{i, j}$ as the full deck group of $R$ associated to the $\operatorname{arc} \sigma$.

Note that $D(R)$ is isomorphic to the symmetric group on $\operatorname{deg}(R)$ symbols. For every $\gamma \in D(R)$ we have that $R(\gamma)=R$ almost everywhere. The group $D(R)$ acts on $L_{1}(\mathbb{C})$ by the push-forward map

$$
\gamma_{*}: f \rightarrow f(\gamma) \gamma^{\prime 2}
$$

with $\left\|\gamma_{*}\right\|_{L_{1}} \leq 1$ for every $\gamma \in D(R)$.

For every subgroup $\Gamma<D(R)$ and $\gamma \in \Gamma$, let $R_{\gamma}=\gamma \circ R \circ \gamma^{-1}=\gamma \circ R$. Define the semigroup

$$
\mathcal{S}(\Gamma)=\left\langle R_{\gamma}\right\rangle_{\gamma \in \Gamma}
$$

Then $\mathcal{S}(\Gamma)$ is a finitely generated semigroup of piecewise holomorphic maps which is RA by Theorem 28, For example if $\Gamma<\operatorname{Deck}(R)$ then $\mathcal{S}(\Gamma)$ consists of rational maps. 
Using the action of $R$ on $L_{1}(\mathbb{C})$ by the Ruelle operator $R_{*}$ we construct the Ruelle representation $\rho: \mathcal{S}(D(R)) \rightarrow \operatorname{End}\left(L_{1}(\overline{\mathbb{C}})\right)$ defined by the formulas on generators:

$$
\rho\left(R_{\gamma}\right)(\phi)=\left(R_{\gamma}\right)_{*}(\phi)=\gamma_{*} \circ R_{*}(\phi)
$$

for $\phi \in L_{1}(\mathbb{C})$. If $A$ is a completely invariant positive Lebesgue measure set, that is $\operatorname{Leb}\left(R^{-1}(A) \backslash A\right)=0$, then $\operatorname{Leb}(\gamma(A) \backslash A)=0$ for $\gamma \in D(R)$, where Leb denotes the Lebesgue measure.

Proposition 39. Let $R$ be a rational map and $A$ be a completely invariant set of positive Lebesgue measure. Assume that A does not support a non-zero invariant Beltrami differential of $R$, then the Ruelle representation of $S(D(R))$ on $L_{1}(A)$ is left amenable.

Proof. The semigroup $S(D(R))$ is RA by Theorem 28, then the space $X_{\rho} \subset$ $L_{\infty}(S(D(R)))$ possesses a right-invariant mean $m$. Recall that $X_{\rho}$ is the closure of the linear span of constant functions together with the space $Y_{\rho}$.

We claim that $\operatorname{ker}(m)$ contains $Y_{\rho}$.

Otherwise, there are two elements $\psi \in L_{1}(A)$ and $\nu \in L_{\infty}(A)$ so that $m\left(\phi_{\psi, \nu}\right) \neq 0$. Then

$$
M(f)=m\left(\phi_{f, \nu}\right)
$$

is a continuous $R_{*}$-invariant functional on $L_{1}(A)$. But $M(\psi)=m\left(\phi_{\psi, \nu}\right) \neq 0$ then by the Riesz representation theorem there exists an invariant Beltrami differential $\mu \neq 0$ which is a contradiction.

Since $X_{\rho}$ and $Y_{\rho}$ are both left-invariant then by the claim every right mean on $X_{\rho}$ is left invariant.

Conversely we have the following theorem.

Theorem 40. Let $R$ be a rational map and $\Gamma<D(R)$ be a transitive subgroup. Assume that $\mathcal{S}(\Gamma)$ is $\rho$-LA, where $\rho$ is the Ruelle representation. Then the following conditions are equivalent.

1. $R_{*}$ has non-zero fixed points in $L_{1}(\mathbb{C})$.

2. $R$ is Möbius conjugated to a flexible Lattés map.

Proof. (1) implies (2). Assume that $R_{*}$ has a non-zero fixed point $f \in L_{1}(\mathbb{C})$. Then by Lemma A in [24, there exists an invariant Beltrami differential $\mu$ with $\mu=\frac{|f|}{f}$ almost everywhere on the support of $f$. We can assume that $R$ acts ergodically on the support of $\mu$. Then the representation $R_{*}: L_{1}(\operatorname{supp}(\mu)) \rightarrow$ $L_{1}(\operatorname{supp}(\mu))$ has fixed point $\alpha \neq 0$ if and only if $\alpha$ is a multiple of $f$. Even more, the Beltrami operator $\left(R_{*}\right)^{*}: L_{\infty}(\operatorname{supp}(\mu)) \rightarrow L_{\infty}(\operatorname{supp}(\mu))$ has a fixed point $\beta \neq 0$ if and only if $\beta$ is a multiple of $\mu$. Then by the separation principle, we conclude that $R_{*}: L_{1}(\operatorname{supp}(\mu)) \rightarrow L_{1}(\operatorname{supp}(\mu))$ is mean-ergodic. 
Even more $R_{*}$ is weakly almost periodic. Indeed since $R_{*}$ is mean-ergodic then the conjugated operator $T(\phi)=\mu R_{*}(\bar{\mu} \phi)$ is also a mean-ergodic operator with the same norm. A straightforward computation shows

$$
T(\phi)(y)=\sum_{R(x)=y} \frac{\phi(x)}{\left|R^{\prime}(x)\right|}=\sum \phi\left(\zeta_{i}(y)\right)\left|\zeta_{i}^{\prime}\right|^{2}(y)
$$

is a positive operator which is almost weakly periodic by Theorem 15, where $\zeta_{i}$ is a complete local system of branches of $R^{-1}$. So $R_{*}$ is weakly almost periodic on $L_{1}(\operatorname{supp}(\mu))$.

The semigroup $\mathcal{S}(\Gamma)$ consists only of iterations of the generators and every generator is conjugated to $R$. Hence $\rho(\mathcal{S}(\Gamma)$ ) also consists only of iteration of the generators $\rho\left(R_{\gamma}\right)$ and each $\rho\left(R_{\gamma}\right)$ is conjugated to $\rho(R)$ where $\rho$ is the Ruelle representation. This implies that $\rho(\mathcal{S}(\Gamma))$ is a weakly almost periodic semigroup of operators on $L_{1}(\operatorname{supp}(\mu))$.

Since $\mathcal{S}(\Gamma)$ is $\rho$-LA, we claim that there exist a functional $\ell \in L_{\infty}^{*}(\operatorname{supp}(\mu))$ which is invariant for the semigroup $(\rho(\mathcal{S}(\Gamma)))^{*}=\left\{t^{*}: t \in \rho(\mathcal{S}(\Gamma))\right\}$.

Indeed if $L$ is a mean we define the functional

$$
\ell(h)=L\left(\phi_{h, f}\right)
$$

for $\phi_{h, f} \in L_{\infty}(\mathcal{S}(\Gamma))$ given by

$$
\phi_{h, f}(g)=\int h \rho(g)(f)|d z|^{2}
$$

where $g \in \mathcal{S}(\Gamma), h \in L_{\infty}(\operatorname{supp}(\mu))$ and $f \in L_{1}(\operatorname{supp}(\mu))$. Since $L$ is left invariant we get $\ell\left(t^{*}(h)\right)=\ell(h)$ for every $t \in \rho(\mathcal{S}(\Gamma))$.

Now we continue the proof of the theorem by standard arguments of functional analysis (see for example [10]). The functional $\ell$ generates a finite complex valued invariant finitely additive measure $\alpha_{\ell}$ defined by the formula

$$
\alpha_{\ell}(A)=\ell\left(\chi_{A}\right)
$$

where $A$ is a measurable subset of $\operatorname{supp}(\mu)$. From the definition follows that $\alpha_{\ell}$ is null on every zero Lebesgue measure subset of $\operatorname{supp}(\mu)$. Next we show that $\alpha_{\ell}$ is a measure absolutely continuous with respect to Lebesgue. It is enough to show that $\alpha_{\ell}$ is a countably additive set function. That is

$$
\alpha_{\ell}\left(\bigcup A_{i}\right)=\sum \alpha_{\ell}\left(A_{i}\right)
$$

for every pairwise disjoint family of measurable subsets of $\operatorname{supp}(\mu)$.

Since $\rho(\mathcal{S}(\Gamma))$ is weakly almost periodic then for every $\epsilon>0$ and every $\beta \in L_{1}(\operatorname{supp}(\mu))$ there exists a $\delta>0$ such that

$$
\int_{B}|t(\beta)| \leq \epsilon
$$

for every $t \in \rho(\mathcal{S}(\Gamma))$ whenever the Lebesgue measure of $B$ is less than $\delta$. 
Let $X \subset \operatorname{supp}(\mu)$ a finite Lebesgue measure set which has a decomposition $X=\bigcup_{i=0}^{\infty} A_{i}$ by a family of pairwise disjoint measurable subsets. Then for every $k$ we have

$$
\left.\alpha_{\ell}(X)=\left(\sum_{i=0}^{k} \alpha_{\ell}\left(A_{i}\right)\right)+\alpha_{\ell}\left(\bigcup_{i=k+1}^{\infty} A_{i}\right)\right)
$$

by finite additivity.

Since $\rho(S(\Gamma))(f)$ is a weakly precompact set, for every $\epsilon>0$ we get a $\delta>0$ so that if $k_{0}$ is such that $\operatorname{Leb}\left(X_{k}\right)<\delta$ for $k>k_{0}$, where $X_{k}=\bigcup_{i=k+1}^{\infty} A_{i}$, then

$$
\left|\alpha_{\ell}\left(X_{k}\right)\right| \leq\left|L\left(\phi_{\chi_{X_{k}}, f}\right)\right| \leq \sup _{g \in \mathcal{S}(\Gamma)} \int_{X_{k}}|\rho(g)(f)||d z|^{2} \leq \epsilon .
$$

Then $\alpha_{\ell}$ is a finite measure which is absolutely continuous with respect to the Lebesgue measure on $\operatorname{supp}(\mu)$. Hence, there exists a non-zero $w \in L_{1}(\operatorname{supp}(\mu))$ so that $\ell(h)=\int h w|d z|^{2}$. Since $\ell$ is $\rho(\mathcal{S}(\Gamma))^{*}$ invariant, then $w$ is $\rho(\mathcal{S}(\Gamma))$ invariant and therefore $w$ is a multiple of $f$.

As $R_{*}(f)=f$, we conclude that $f$ is a fixed point for $\gamma_{*}$, with $\gamma \in \Gamma$. Since $\Gamma$ is transitive, we can choose $d=\operatorname{deg}(R)$ elements $\gamma_{1}, \ldots, \gamma_{d} \in \Gamma$ so that for every fixed branch $\zeta_{i}$ of $R^{-1}$ on $\overline{\mathbb{C}} \backslash \sigma$ we have that the collection $\left\{\gamma_{j} \circ \zeta_{i}\right\}$ forms a complete collection of branches of $R^{-1}$ on $\overline{\mathbb{C}} \backslash \sigma$. Therefore,

$$
d w\left(\zeta_{i}\right)\left(\zeta_{i}^{\prime}\right)^{2}=\sum_{j}\left(\gamma_{j}\right)_{*}(w) \circ\left(\zeta_{i}\right)\left(\zeta_{i}^{\prime}\right)^{2}=\sum w\left(\zeta_{j}\right)\left(\zeta_{j}^{\prime}\right)^{2}=w .
$$

Then for every $z \in R^{-1}(\overline{\mathbb{C}} \backslash \sigma)$ we have

$$
\frac{w(R(z)) R^{\prime 2}(z)}{\operatorname{deg}(R)}=w(z) .
$$

Hence $|w|$ defines a continuous functional on $C(\overline{\mathbb{C}})$, via $\phi \mapsto \int_{\mathbb{C}} \phi|w||d z|^{2}$, which is invariant under Lyubich operator $L_{R}$ and so by Theorem 7 and Lemma 18 it is the density of the measure of maximal entropy for $R$. Thus, the map $R$ has maximal entropy measure absolutely continuous with respect to Lebesgue. By Zdunik's Theorem (see [32]) the map $R$ is an exceptional map. Since $\frac{|f|}{f}$ is an invariant Beltrami differential for $R$, then $R$ is a flexible Lattès map.

Now (2) implies (1). If $R$ is a flexible Lattès map, then again by Zdunik's Theorem, the measure $m_{R}$ of maximal entropy of $R$ is absolutely continuous with respect to Lebesgue. Then $d m_{R}=\omega|d z|^{2}$ with $\omega \in L_{1}(\overline{\mathbb{C}}), \omega>0$ and

$$
\frac{\omega(R)\left|R^{\prime}\right|^{2}}{\operatorname{deg}(R)}=\omega
$$

almost everywhere. On the other hand, $R$ has non-zero invariant Beltrami differential $\mu$, hence the function $\bar{\mu} \omega$ is fixed by $R_{*}$, and we are done. 


\section{References}

[1] P. Atela and J. Hu, Commuting polynomials and polynomials with same Julia set, Internat. J. Bifur. Chaos Appl. Sci. Engrg. 6 (1996), no. 12A, $2427-2432$.

[2] A. F. Beardon, Symmetries of Julia sets, Bull. London Math. Soc. 22 (1990), no. 6, 576-582.

[3] J.P. Bell, K. Huang, W. Peng, and T.J. Tucker, A Tits alternative for rational functions, 2021.

[4] C. Cabrera and P. Makienko, On the fixed points of the Ruelle operator, Ergodic Theory Dynam. Systems 41 (2021), no. 4, 1025-1063.

[5] C. Cabrera, P. Makienko, and P. Plaumann, Semigroup representations in holomorphic dynamics, Discrete Contin. Dyn. Syst. 33 (2013), no. 4, 1333-1349.

[6] M. Day, Amenable semigroups, Illinois J. Math. 1 (1957), 509-544.

[7] M. M. Day, Means for the bounded functions and ergodicity of the bounded representations of semi-groups, Trans. Amer. Math. Soc. 69 (1950), 276291.

[8] M. M. Day, Semigroups and amenability, Semigroups (Proc. Sympos., Wayne State Univ., Detroit, Mich., 1968), Academic Press, New York, 1969 , pp. $5-53$.

[9] A. Douady, Systèmes dynamiques holomorphes, Bourbaki seminar, Vol. 1982/83, Astérisque, vol. 105, Soc. Math. France, Paris, 1983, pp. 39-63.

[10] N. Dunford and J.T. Schwartz, Linear operators. Part I, Wiley Classics Library, John Wiley \& Sons, Inc., New York, 1988, General theory, With the assistance of William G. Bade and Robert G. Bartle, Reprint of the 1958 original, A Wiley-Interscience Publication. MR 1009162 (90g:47001a)

[11] A. Eremenko, Some functional equations connected with the iteration of rational functions, Algebra i Analiz 1 (1989), no. 4, 102-116.

[12] _ On the characterization of a Riemann surface by its semigroup of endomorphisms, Trans. Amer. Math. Soc. 338 (1993), no. 1, 123-131.

[13] D. Ghioca, T. J. Tucker, and M. E. Zieve, Intersections of polynomials orbits, and a dynamical Mordell-Lang conjecture, Invent. Math. 171 (2008), no. $2,463-483$.

[14] E. Granirer, On amenable semigroups with a finite-dimensional set of invariant means. II, Illinois J. Math. 7 (1963), 49-58. 
[15] A. Hinkkanen and G. J. Martin, The dynamics of semigroups of rational functions. I, Proc. London Math. Soc. (3) 73 (1996), no. 2, 358-384.

[16] M. Klawe, Semidirect product of semigroups in relation to amenability, cancellation properties, and strong Følner conditions, Pacific J. Math. 73 (1977), no. 1, 91-106.

[17] I. Kornfeld and M. Lin, Weak almost periodicity of $L_{1}$ contractions and coboundaries of non-singular transformations, Studia Math. 138 (2000), no. 3, 225-240. MR 1758856 (2001b:28023)

[18] U. Krengel, Ergodic theorems, de Gruyter Studies in Mathematics, vol. 6, Walter de Gruyter \& Co., Berlin, 1985.

[19] G. Levin and F. Przytycki, When do two rational functions have the same Julia set?, Proc. Amer. Math. Soc. 125 (1997), no. 7, 2179-2190.

[20] G. M. Levin, Symmetries on a Julia set, Dynamical systems and statistical mechanics (Moscow, 1991), Adv. Soviet Math., vol. 3, Amer. Math. Soc., Providence, RI, 1991, pp. 131-142.

[21] M. Lyubich, The measure of maximal entropy of a rational endomorphism of a Riemann sphere, Funktsional. Anal. i Prilozhen. 16 (1982), no. 4, 78-79.

[22] — Entropy properties of rational endomorphisms of the Riemann sphere, Ergodic Theory Dynam. Systems 3 (1983), no. 3, 351-385.

[23] _ Dynamics of the rational transforms; the topological picture, Russian Math. Surveys (1986).

[24] P. Makienko, Remarks on the Ruelle operator and the invariant line fields problem: II, Ergodic Theory and Dynamical Systems 25 (2005), no. 05, $1561-1581$.

[25] O. Ore, Linear equations in non-commutative fields, Ann. of Math. (2) 32 (1931), no. 3, 463-477.

[26] F. Pakovich, On amenable semigroups of rational functions, 2020.

[27] - On rational functions sharing the measure of maximal entropy, Arnold Math. J. 6 (2020), no. 3-4, 387-396.

[28] Fedor Pakovich, Commuting rational functions revisited, Ergodic Theory Dynam. Systems 41 (2021), no. 1, 295-320.

[29] J. F. Ritt, Permutable rational functions, Trans. Amer. Math. Soc. 25 (1923), no. 3, 399-448.

[30] — Equivalent rational substitutions, Trans. Amer. Math. Soc. 26 (1924), no. 2, 221-229. 
[31] Hexi Ye, Rational functions with identical measure of maximal entropy, Adv. Math. 268 (2015), 373-395.

[32] A. Zdunik, Parabolic orbifolds and the dimension of the maximal measure for rational maps, Invent. Math. 99 (1990), no. 3, 627-649. MR 1032883 (90m:58120)

C. Cabrera Unidad Cuernavaca del Instituto de Matemáticas. UnAM, México

E-mail: carloscabrerao@im.unam.mx

P. Makienko Unidad Cuernavaca del Instituto de Matemáticas. unam, México

E-mail: makienko@im.unam.mx 